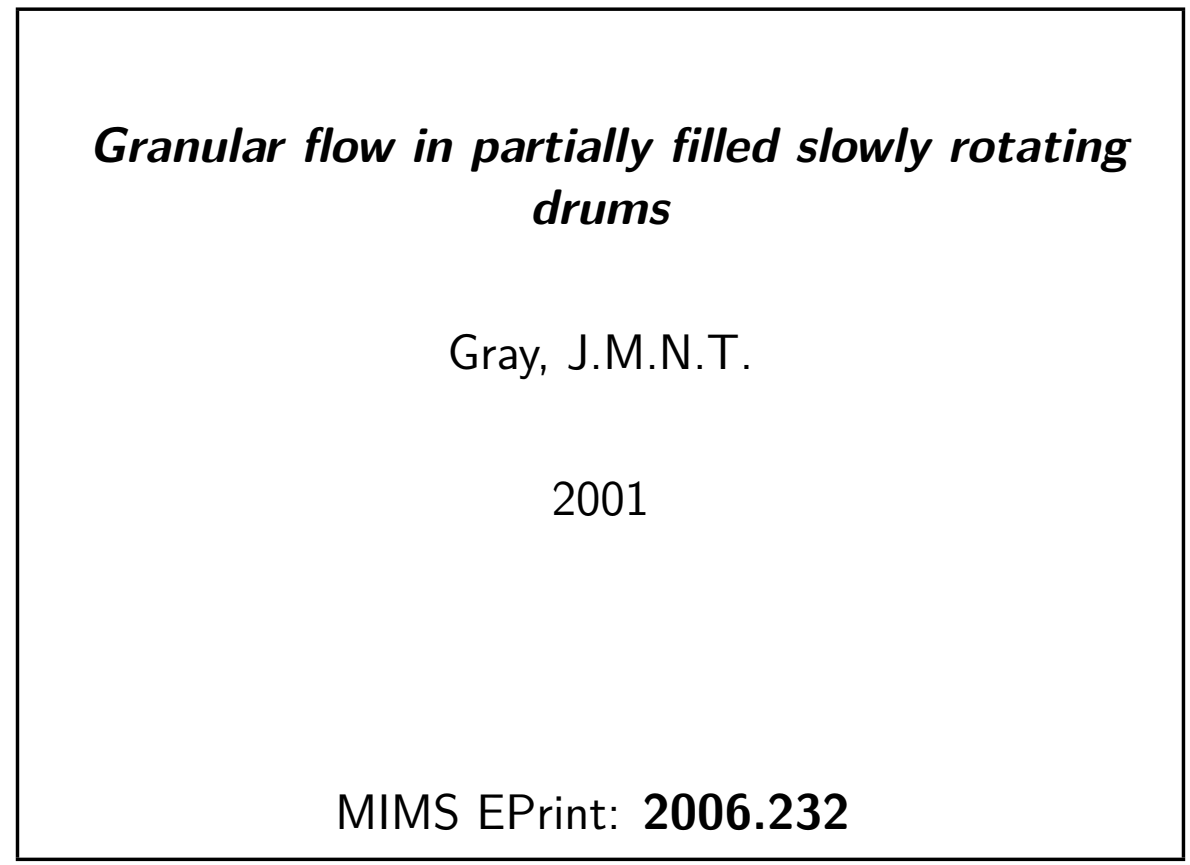

Manchester Institute for Mathematical Sciences

School of Mathematics

The University of Manchester

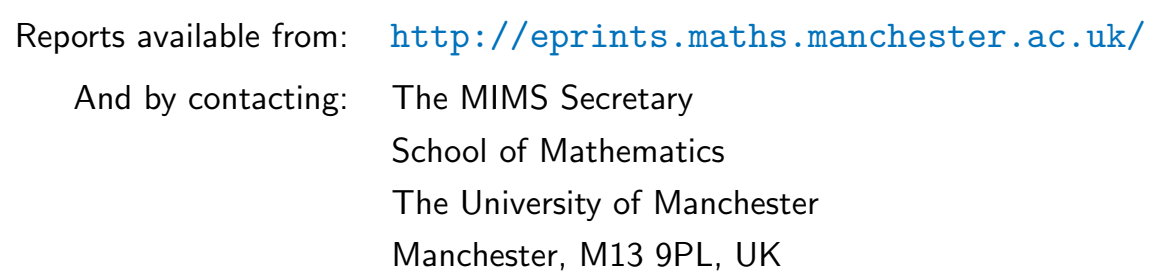

ISSN 1749-9097 


\title{
Granular flow in partially filled slowly rotating drums
}

\author{
By J. M. N. T. GRA Y \\ Institut für Mechanik, Technische Universität Darmstadt, 64289 Darmstadt, Germany \\ and Department of Mathematics, University of Manchester, Manchester M13 9PL UK
}

(Received 3 January 2000 and in revised form 3 January 2001)

In many industrial processes granular materials are mixed together in partially filled slowly rotating drums. In this paper a general theoretical framework is developed for the quasi-two-dimensional motion of granular material in a rotating drum. The key assumption is that the body can be divided into a fluid-like and a solid-like region, that are separated by a non-material singular surface at which discontinuities occur. Experiments show that close to the free surface there is a thin rapidly moving fluidlike avalanche that flows downslope, and beneath it there is a large region of slowly rotating solid-like material. The solid region provides a net transport of material upslope and there is strong mass transfer between the two regions. In the theory the avalanche is treated as a shallow incompressible Mohr-Coulomb or inviscid material sliding on a moving bed at which there is erosion and deposition. The solid is treated as a rigid rotating body, and the two regions are coupled together using a mass jump condition. The theory has the potential to model time-dependent intermittent flow with shock waves, as well as steady-state continuous flow. An exact solution for the case of steady continuous flow is presented. This demonstrates that when the base of the avalanche lies above the axis of revolution a solid core develops in the centre of the drum. Experiments are presented to show how a mono-disperse granular material mixes in the drum, and the results are compared with the predictions using the exact solution.

\section{Introduction}

\subsection{Intermittent and continuous flow}

In many food manufacturing and industrial processes granular materials are often placed into a drum, pivoted so that its axis of rotational symmetry lies horizontally, and slowly rotated to try to mix the grains into a consistent blend (e.g. Ullrich 1969; Metcalfe et al. 1995; McCarthy et al. 1996; Ristow 1996; de Gennes 1999). In all these flows the motion is characterized by a thin rapidly moving fluid-like avalanche of grains close to the free surface and a large solid-like region, beneath it, which slowly rotates about the axis of revolution.

At slow rotation speeds there are essentially two flow regimes: intermittent flow and continuous flow (Rajchenbach 1990). In the intermittent flow regime, which is sometimes called avalanching flow, there are discrete avalanche events that come to rest before the next event starts, whereas in the continuous regime granular material is continually flowing downslope in a quasi-steady state. In this paper the term avalanche will be used to refer to both the intermittent and continuous regimes, since they are both described by a single system of avalanche equations. 
In intermittent flow, the granular material is slowly rotated until the free surface reaches the maximum angle of repose. Failure then occurs along an internal slip line and and an avalanche is released, which flows rapidly downslope. If the flow is supercritical a shock wave (Gray \& Hutter 1997) is generated when the avalanche front reaches the drum wall. The shock wave propagates upslope in the opposite direction to the flow, bringing the avalanche to rest. The free surface inclination angle then lies below the maximum angle of repose, and the material that is deposited is absorbed into the solid body region. Further rotation induces another avalanche and the process is repeated. The solid deposit is slowly rotated until it eventually reaches the top of the avalanche again, providing a net transport of material upslope. The intermittent flow regime is therefore characterized by discrete avalanche events and shock wave propagation. The picture is complicated further by mass transfer between the avalanche and the underlying solid region whilst the avalanche is in motion. Intermittent flow occurs at rotation periods above about $100 \mathrm{~s}$ per revolution. The exact range for this flow regime is dependent on the shape and surface roughness of the grains, the size of the container and whether slip occurs between the granular material and the drum wall.

Continuous flow occurs in the range 10-100s per revolution in the experiments presented here. In this regime the free surface is steady, there is continuous avalanching flow and continuous mass transfer with the underlying solid body region. Material is fed to the top half of the avalanche by slow rotation in the solid body region, the avalanche then transports this material rapidly downslope, where it is reabsorbed into the solid body region and transported to the top again. All processes take place continuously and this flow configuration is stable in a wide range of rotation periods. As the rotation period is decreased below about $10 \mathrm{~s}$ per revolution the avalanche has difficulty in transporting the large volume of granular material that is supplied along the upper half of the solid interface rapidly enough downslope. The free surface therefore steepens up to angles that are significantly above the angle of repose, and intermittent breaking waves develop. At even faster rotation periods the centrifugal forces become important and voids open up within the solid region of the granular material (e.g. Gray \& Hutter 1998).

The differences between continuous and intermittent flow become immediately apparent when a mixture of small dark and large white particles is placed into a thin drum and rotated (Gray \& Hutter 1997, 1998).

\subsection{Solid- and fluid-like regions}

The key assumption in the theoretical treatment presented here is that the fluid-like avalanche and the solid-like region can be treated as separate bodies with a nonmaterial singular surface between them, at which the field variables are discontinuous. This is a mathematical idealization and in practice there is a thin layer between the two regions, where there are large gradients of the velocity and density. However, it appears to be a very good approximation as the subsequent work will show. The dominant physical processes are the dynamics of the avalanche and the mass transfer between the avalanche and the solid region beneath it. The constitutive properties of the solid granular material are therefore kept as basic as possible.

Granular avalanches not only take place in rotating drums, but are abundant in our natural environment on a wide range of length scales. In the kitchen or on the dining table sugar and salt can form avalanches on the scale of a few centimetres if the surface layer is tilted so that it becomes unstable. Avalanches also take place at the free surface of stock piles, hopper flows and silos. They can also be observed on 
the geophysical scale as landslides, debris flows, rock-falls and snow (slab) avalanches. Yet despite the enormous difference in length scales the dominant physical mechanisms that drive the flow are similar. The motivation for the avalanche model derived in this paper comes from a continuum theory that was developed by Savage \& Hutter (1989) for modelling snow slab avalanches.

\subsection{The Savage-Hutter avalanche theory}

The original Savage \& Hutter (1989) theory for plane two-dimensional flow assumed that the avalanche was a shallow incompressible Mohr-Coulomb material that slid down a rigid impenetrable surface inclined at an angle $\zeta_{0}$ to the horizontal. The leading-order mass and momentum equations were integrated through the avalanche depth to obtain a one-dimensional theory along the flow direction. The model enabled the avalanche thickness and the depth-averaged velocity to be computed, once the basal topography was prescribed together with suitable initial values and boundary conditions. This model has been particularly effective at modelling shallow granular flows both in nature and in laboratory experiments. Savage \& Hutter (1991) extended their theory to allow the flow on more complicated slopes to be modelled by introducing a slope-fitted curvilinear coordinate system. This allowed the inclination angle of the slope $\zeta$ to change as a function of the distance down the slope. Laboratory experiments on an exponentially curved chute (Hutter \& Koch 1991) and on concave and convex chutes (Greve \& Hutter 1993; Hutter et al. 1995) showed that the model was in very good agreement. More recently the theory has been generalized to three dimensions (Hutter et al. 1993; Greve, Koch \& Hutter 1994; Gray, Weiland \& Hutter 1999) to model flows over complex three-dimensional topography. These models have been very effective at modelling the laboratory flows on inclined planes, and on chutes both with and without lateral curvature (Wieland, Gray \& Hutter 1999; Koch, Greve \& Hutter 1994).

Here a theory is derived for the two-dimensional plane motion of shallow granular avalanches flowing on a moving bed at which there is erosion and deposition of the granular material. The model differs in four key points to the model of Savage \& Hutter (1989). First, the three-dimensional conservative form of the momentum balance is used to derive two conservative two-dimensional depth-integrated momentum balances, in contrast to the non-conservative momentum balances derived by Savage \& Hutter (1989). While this difference is not important for classical smooth solutions (e.g. Hutter \& Nohguchi 1990; Hutter \& Greve 1993; Hutter 1996) it is important to use this form when constructing shock-capturing numerical methods for non-classical solutions with shocks (e.g. Yee 1989; Nessyahu \& Tadmor 1990; Tóth \& Odstrčil 1996; Jiang \& Tadmor 1997). This is essential for obtaining the correct shock speeds in the intermittent flow regime. The second and most important difference is that surface and basal erosion and deposition are considered. Thirdly the basal topography is allowed to have a non-zero velocity, and finally both Mohr-Coulomb and inviscid constitutive models are considered.

\section{Avalanches with erosion and deposition}

\subsection{Conservation equations and boundary conditions}

Granular materials exhibit dilatancy effects during plastic yield. Once failure has occurred and the material is fluidized, however, it is reasonable to assume that it is incompressible with constant uniform density, $\rho_{0}$. The conservative form of the mass 
and momentum balances reduce to

$$
\begin{gathered}
\nabla \cdot \boldsymbol{u}=0, \\
\partial_{t}\left(\rho_{0} \boldsymbol{u}\right)+\nabla \cdot\left(\rho_{0} \boldsymbol{u} \otimes \boldsymbol{u}\right)=-\nabla \cdot \boldsymbol{p}+\rho_{0} \boldsymbol{g},
\end{gathered}
$$

where $\nabla$ is the gradient operator, $\boldsymbol{u}$ is the velocity, $\partial_{t}$ indicates differentiation with respect to time, $\otimes$ is the tensor (or dyadic) product, $\boldsymbol{p}$ is the pressure tensor (the negative Cauchy stress) and $\boldsymbol{g}$ is the gravitational acceleration.

The avalanche lies between an upper non-material free surface, $F^{s}(\boldsymbol{x}, t)=0$, and a basal non-material interface, $F^{b}(\boldsymbol{x}, t)=0$. These surfaces are defined so that the unit normals at the free surface $\boldsymbol{n}^{s}=\nabla F^{s} /\left|\nabla F^{s}\right|$ and base $\boldsymbol{n}^{b}=\nabla F^{b} /\left|\nabla F^{b}\right|$ point outward from the avalanche. The superscripts $s$ and $b$ indicate that a variable is evaluated at the surface or the basal interface, respectively. Assuming that the non-material surface and basal interfaces have velocities $\boldsymbol{v}^{s}$ and $\boldsymbol{v}^{b}$ the kinematic boundary conditions are

$$
\begin{array}{ll}
F^{s}(\boldsymbol{x}, t)=0: & \partial_{t} F^{s}+\boldsymbol{v}^{s} \cdot \nabla F^{s}=0, \\
F^{b}(\boldsymbol{x}, t)=0: & \partial_{t} F^{b}+\boldsymbol{v}^{b} \cdot \nabla F^{b}=0 .
\end{array}
$$

The avalanche is traction free at the surface and slides over the underlying material at the base generating a shear traction that is proportional to the basal pressure, i.e. Coulomb dry friction is assumed

$$
\begin{gathered}
F^{s}(\boldsymbol{x}, t)=0: \quad \boldsymbol{p}^{s} \boldsymbol{n}^{s}=\mathbf{0}, \\
F^{b}(\boldsymbol{x}, t)=0: \quad \boldsymbol{p}^{b} \boldsymbol{n}^{b}=\left(\boldsymbol{u}^{r} /\left|\boldsymbol{u}^{r}\right|\right) \tan \delta\left(\boldsymbol{n}^{b} \cdot \boldsymbol{p}^{b} \boldsymbol{n}^{b}\right)+\boldsymbol{n}^{b}\left(\boldsymbol{n}^{b} \cdot \boldsymbol{p}^{b} \boldsymbol{n}^{b}\right),
\end{gathered}
$$

where $\delta$ is the basal angle of friction and the factor $\left(\boldsymbol{u}^{r} /\left|\boldsymbol{u}^{r}\right|\right)$ ensures that the Coulomb friction opposes the avalanche motion. The relative velocity $\boldsymbol{u}^{r}=\boldsymbol{u}^{b+}-\boldsymbol{u}^{b-}$ is the velocity difference between the base of the avalanche, $\boldsymbol{u}^{b+}$, and the basal topography, $\boldsymbol{u}^{b-}$. This definition differs from that of Savage \& Hutter (1989) to reflect the fact that the basal topography can have a non-zero velocity component parallel to the interface. Additional effects such as air drag, rate-dependent basal drags and momentum thrust terms due to mass interaction are neglected.

A fixed Cartesian coordinate system $o x z$ with origin $o$ is defined, so that the $x$-axis lies approximately parallel to the bed interface, $F^{b}$, and points in the downslope direction. The $x$-axis is, therefore, inclined at an angle $\zeta$ to the horizontal and the $z$-axis is chosen along the upward pointing normal. The coordinate system is illustrated in figure 1 . The velocity $\boldsymbol{u}$ has components $u$ and $w$ in the downslope and normal directions, respectively, and the symmetric pressure tensor has components $p_{x x}, p_{x z}, p_{z z}$.

In typical rotating drum experiments and in geophysical flows the granular avalanche depth, $H$, is much smaller than the avalanche length, $R$. It is convenient to introduce non-dimensional variables to reflect the shallowness of the granular avalanche

$$
\left.\begin{array}{l}
(x, z, t)=\left(R \tilde{x}, H \tilde{z},(R / g)^{1 / 2} \tilde{t}\right), \\
\left(u, w, v_{n}^{s}, v_{n}^{b}\right)=(R g)^{1 / 2}\left(\tilde{u}, \varepsilon \tilde{w}, \varepsilon \tilde{v}_{n}^{s}, \varepsilon \tilde{v}_{n}^{b}\right), \\
\left(p_{x x}, p_{z z}\right)=\rho_{0} g H\left(\tilde{p}_{x x}, \tilde{p}_{z z}\right), \\
\left(p_{x z}\right)=\rho_{0} g H \mu\left(\tilde{p}_{x z}\right),
\end{array}\right\}
$$

where the tildes denote non-dimensional variables, $g$ is the constant of gravitational acceleration and $\varepsilon=H / R \ll 1$ is the aspect ratio. Note, that the Coulomb friction law (2.6) implies that the magnitude of the shear stress is equal to the magnitude of the basal pressure, $\rho_{0} g H$, multiplied by Coulomb friction coefficient $\mu=\tan \delta_{0}$. 


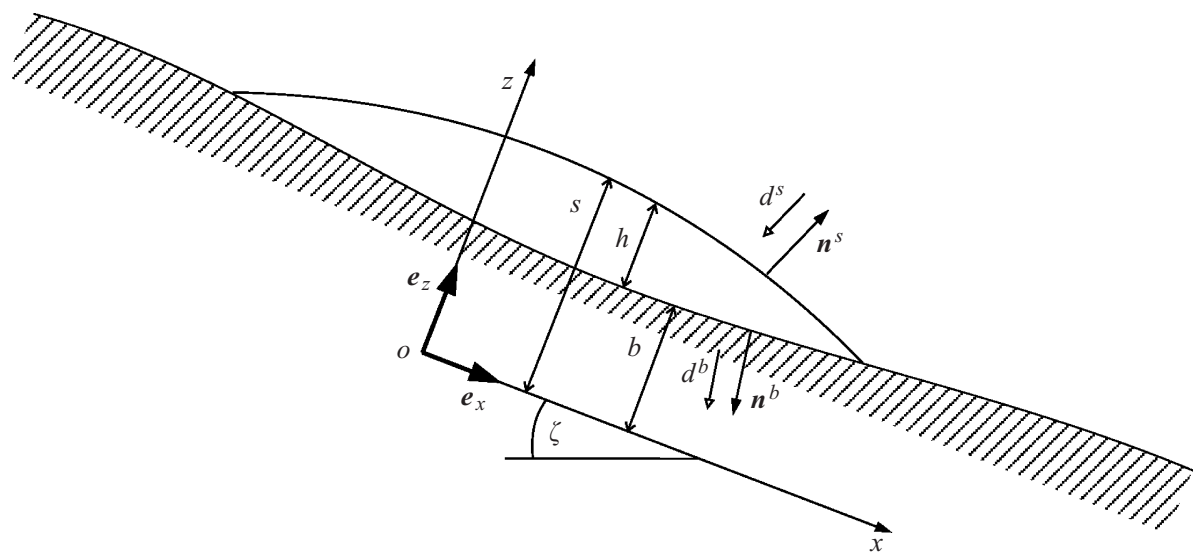

FiguRE 1. A sketch of the avalanche and basal topography. A fixed Cartesian coordinate system $o x z$ is defined with the $x$-axis pointing in the downslope direction and inclined at an angle $\zeta$ to the horizontal. The avalanche free surface lies at $z=s(x, t)$ and the base at $z=b(x, t)$. The avalanche thickness $h(x, t)$ is measured parallel to the $z$-axis. The accumulation rate $d^{s}$ and the deposition rate $d^{b}$ are the equivalent volumes of granular material that are either deposited on the avalanche free surface, or eroded from the avalanche base, respectively, per unit area per unit time.

Applying the scalings (2.7) the non-dimensional mass balance (2.1) is

$$
\partial_{x} u+\partial_{z} w=0,
$$

where the tildes are now dropped, and $\partial_{x}$ and $\partial_{z}$ denote differentiation with respect to $x$ and $z$, respectively. The downslope and normal components of the momentum balance (2.2) are

$$
\begin{gathered}
\partial_{t} u+\partial_{x}\left(u^{2}\right)+\partial_{z}(u w)=\sin \zeta-\varepsilon \partial_{x}\left(p_{x x}\right)-\mu \partial_{z} p_{x z}, \\
\varepsilon\left\{\partial_{t} w+\partial_{x}(u w)+\partial_{z}\left(w^{2}\right)\right\}=-\cos \zeta-\varepsilon \mu \partial_{x}\left(p_{x z}\right)-\partial_{z} p_{z z} .
\end{gathered}
$$

The avalanche free surface, $F^{s}=z-s(x, t)=0$, and the basal interface $F^{b}=$ $b(x, t)-z=0$, are defined by their heights above the $z=0$ plane. These definitions ensure that the surface and basal unit normals point outwards from the avalanching material and are given by

$$
\psi^{s} \boldsymbol{n}^{s}=-\varepsilon \partial_{x} \boldsymbol{s} \boldsymbol{e}_{x}+\boldsymbol{e}_{z}, \quad \psi^{b} \boldsymbol{n}^{b}=\varepsilon \partial_{x} b \boldsymbol{e}_{x}-\boldsymbol{e}_{z},
$$

where $\boldsymbol{e}_{x}$ and $\boldsymbol{e}_{z}$ are unit vectors in the downslope and normal directions, respectively, and the normalization factors $\psi^{s}=\left\{1+\varepsilon^{2}\left(\partial_{x} s\right)^{2}\right\}^{1 / 2}$ and $\psi^{b}=\left\{1+\varepsilon^{2}\left(\partial_{x} b\right)^{2}\right\}^{1 / 2}$. The surface and basal interfaces are modified by accumulation and deposition. Let the normal accumulation rate, $d^{s}$, be the equivalent volume of granular material deposited on the avalanche free surface per unit area per unit time. Then the velocity of the interface $\boldsymbol{v}^{s}=\boldsymbol{u}^{s}+d^{s} \boldsymbol{n}^{s}$. Similarly, if the normal deposition rate, $d^{b}$, is the equivalent volume of granular material deposited at the avalanche base per unit area per unit time, the velocity of the basal interface $\boldsymbol{v}^{b}=\boldsymbol{u}^{b}-d^{b} \boldsymbol{n}^{b}$. It follows that the surface and basal kinematic conditions (2.3) and (2.4) are

$$
\begin{array}{cc}
z=s(x, t): & \partial_{t} s+u^{s} \partial_{x} s-w^{s}=\psi^{s} d^{s}, \\
z=b(x, t): & \partial_{t} b+u^{b} \partial_{x} b-w^{b}=\psi^{b} d^{b} .
\end{array}
$$

There are many situations in which surface accumulation or basal deposition can 
occur, for instance when a heap of granular material is built up on a horizontal plane. The granular material that is poured onto the top of the pile provides a source of surface accumulation to the avalanche and as the granular material comes to rest it is deposited into the solid body region of granular material beneath it. This deposition mechanism can equivalently be viewed as a moving phase boundary between solid and fluid-like regions.

At the free surface the traction-free boundary condition (2.5) has downslope and normal components

$$
\begin{aligned}
& -\varepsilon p_{x x}^{s} \partial_{x} s+\mu p_{x z}^{s}=0, \\
& -\varepsilon \mu p_{z x}^{s} \partial_{x} s+p_{z z}^{s}=0,
\end{aligned}
$$

and the Coulomb friction law (2.6) has downslope and normal traction components

$$
\begin{aligned}
\varepsilon p_{x x}^{b} \partial_{x} b-\mu p_{x z}^{b} & =\left(\boldsymbol{n}^{b} \cdot \boldsymbol{p}^{b} \boldsymbol{n}^{b}\right)\left(\psi^{b}\left(u^{r} /\left|\boldsymbol{u}^{r}\right|\right) \tan \delta+\varepsilon \partial_{x} b\right), \\
\varepsilon \mu p_{z x}^{b} \partial_{x} b-p_{z z}^{b} & =\left(\boldsymbol{n}^{b} \cdot \boldsymbol{p}^{b} \boldsymbol{n}^{b}\right)\left(\psi^{b}\left(\varepsilon w^{r} /\left|\boldsymbol{u}^{r}\right|\right) \tan \delta-1\right) .
\end{aligned}
$$

Note that as a first approximation the accumulation and deposition is assumed to have no effect on the mechanical boundary conditions at the surface and base of the avalanche. In general more complex boundary conditions may be necessary, which take account of suction effects or the 'granular temperature' of the material.

\subsection{Integration through the avalanche depth}

Following Savage \& Hutter (1989) the governing equations are now integrated through the avalanche depth to obtain a simplified depth-averaged theory in which one spatial dimension is removed from the problem. The avalanche thickness $h$ is equal to the difference between the free surface height, $s(x, t)$, and the height of the basal interface, $b(x, t)$,

$$
h=s-b,
$$

and the depth-averaged value $\bar{f}$ of a function $f$ is

$$
\bar{f}=\frac{1}{h} \int_{b}^{s} f \mathrm{~d} z .
$$

Integrating the mass balance (2.8) through the avalanche depth and using Leibnitz's rule to interchange the order of integration and differentiation, it follows that

$$
\partial_{x}(h \bar{u})-\left[u \partial_{x} z-w\right]_{b}^{s}=0,
$$

where the square bracket is the difference between the surface and basal values of the enclosed function, i.e. $[f]_{b}^{s}=f^{s}-f^{b}$. Substituting the kinematic boundary conditions (2.12) and (2.13), and using (2.18), the depth-integrated mass balance is

$$
\partial_{t} h+\partial_{x}(h \bar{u})=\psi^{s} d^{s}-\psi^{b} d^{b} .
$$

Integrating the downslope momentum balance (2.9) through the avalanche depth using Leibnitz's rule implies

$$
\partial_{t}(h \bar{u})+\partial_{x}\left(h \overline{u^{2}}\right)-\left[u\left(\partial_{t} z+u \partial_{x} z-w\right)\right]_{b}^{s}=h \sin \zeta+\varepsilon \partial_{x}\left(h \overline{p_{x x}}\right)-\left[\varepsilon p_{x x} \partial_{x} z-\mu p_{x z}\right]_{b}^{s} .
$$

Substituting the kinematic conditions (2.12) and (2.13) and the downslope traction conditions (2.14) and (2.16) into the square bracketed terms gives the depth-integrated 
downslope momentum balance

$$
\begin{aligned}
\partial_{t}(h \bar{u})+\partial_{x}\left(h \overline{u^{2}}\right)-\left(u^{s} \psi^{s} d^{s}-u^{b} \psi^{b} d^{b}\right) \\
=h \sin \zeta-\left(\psi^{b}\left(u^{r} /\left|\boldsymbol{u}^{r}\right|\right) \tan \delta+\varepsilon \partial_{x} b\right)\left(\boldsymbol{n}^{b} \cdot \boldsymbol{p}^{b} \boldsymbol{n}^{b}\right)-\varepsilon \partial_{x}\left(h \overline{p_{x x}}\right) .
\end{aligned}
$$

\subsection{Order of magnitude estimates}

The shallowness of the granular avalanche is now exploited by making a long-wave approximation based on the small parameter $\varepsilon=H / L$. Since the friction coefficient $\mu=\tan \delta_{0}$ is less than order unity, but greater than order $\varepsilon$, it is convenient to make the approximation that $\mu=O\left(\varepsilon^{\gamma}\right)$, for some exponent $\gamma \in(0,1)$. To accurately describe the flow of granular avalanches, terms of order $\varepsilon$ must be retained in the theory, but terms of order $\varepsilon^{1+\gamma}$ can be discarded. The effect of this scaling is to retain longitudinal pressure gradients, but to neglect order- $\varepsilon$ pressure corrections to the Coulomb dry friction law.

Integrating the normal component of the momentum balance (2.10) with respect to $z$ and applying the free surface boundary condition (2.15), it follows that

$$
p_{z z}=(s-z) \cos \zeta+O(\varepsilon),
$$

and at the base

$$
p_{z z}^{b}=h \cos \zeta+O(\varepsilon) .
$$

Hence, the basal normal pressure $\boldsymbol{n}^{b} \cdot \boldsymbol{p}^{b} \boldsymbol{n}^{b}=h \cos \zeta+O(\varepsilon)$ in the Coulomb dry friction law. It follows that to order $\varepsilon^{1+\gamma}$ the depth-integrated mass and downslope momentum balances reduce to

$$
\begin{gathered}
\partial_{t} h+\partial_{x}(h \bar{u})=d^{s}-d^{b}, \\
\partial_{t}(h \bar{u})+\partial_{x}\left(h \overline{u^{2}}\right)-\left(u^{s} d^{s}-u^{b} d^{b}\right)=h D-\varepsilon \partial_{x}\left(h \overline{p_{x x}}\right)-\varepsilon h \cos \zeta \partial_{x} b,
\end{gathered}
$$

where the net driving force

$$
D=\cos \zeta\left(\tan \zeta-\left(u^{r} /\left|u^{r}\right|\right) \tan \delta\right),
$$

is the difference between the gravitational acceleration and the Coulomb basal friction.

\subsection{Constitutive properties}

To complete the theory, a constitutive relation is required for the depth-averaged longitudinal stress, $\overline{p_{x x}}$, in the fluid-like granular flow regime. This is still a subject of current research, and two relations will be considered in this paper.

Following Savage \& Hutter (1989), who assumed that the avalanche behaved as a Mohr-Coulomb-type material at yield, it is assumed that on each plane element the normal pressure $N$ and the tangential shear stress $\boldsymbol{S}$ are related by a Mohr-Coulomb yield criterion

$$
|\boldsymbol{S}|=N \tan \phi,
$$

where $\phi$ is the internal angle of friction. At the base of the avalanche the yield criterion (2.29) and the sliding law (2.6) must be satisfied simultaneously. The basal stress state, $\left(p_{z z}^{b},-p_{z z}^{b} \tan \delta\right)$, must therefore lie on a Mohr circle of stress that is tangent to the yield lines $|\boldsymbol{S}|=N \tan \phi$. A Mohr diagram showing the yield criterion and sliding law is illustrated in Savage \& Hutter (1989). There are two solution circles, associated with 'active' and 'passive' states of the system.

Using the Mohr circle diagram Savage \& Hutter (1989) used elementary trigonometrical relations to show that the basal longitudinal and normal pressures were 
linearly related,

$$
p_{x x}^{b}=K p_{z z}^{b},
$$

by an earth pressure coefficient, $K$. In the active and passive states this takes the values

$$
K_{a c t / p a s}=2 \sec ^{2} \phi\left(1 \mp\left\{1-\cos ^{2} \phi \sec ^{2} \delta\right\}^{1 / 2}\right)-1,
$$

which are real valued provided $\delta \leqslant \phi$. The active and passive states are associated with extensive $\left(\partial_{x} u \geqslant 0\right)$ and compressive $\left(\partial_{x} u<0\right)$ motions, respectively. A smooth transition for low strain rates (e.g. Tai \& Gray 1998) can be adopted to avoid a jump in longitudinal stress at $\partial_{x} u=0$. Savage \& Hutter (1989) implicitly assumed that for suitably thin avalanches, equations (2.30) and (2.31) hold through the avalanche depth to order $\varepsilon^{1+\gamma}$. Substituting (2.24) into (2.30) and integrating over the avalanche depth implies that

$$
h \overline{p_{x x}}=K \cos \zeta h^{2} / 2+O(\varepsilon),
$$

which provides an expression for the longitudinal pressure.

The assumption that a Mohr-Coulomb yield criterion is appropriate for rapidly flowing granular material is rather strong, as the material may be well beyond the point of yield. An inviscid fluid model, with an isotropic pressure distribution

$$
p_{x x}=p_{z z}
$$

is therefore also considered as an alternative method of closure. Integrating over the avalanche depth, implies that the longitudinal pressure satisfies the simpler relation

$$
h \overline{p_{x x}}=\cos \zeta h^{2} / 2+O(\varepsilon) .
$$

A comparison with (2.32) shows that this is equivalent to assuming that the earth pressure coefficient $K=1$, and that there is no jump in the longitudinal stress. For extensive flow it may be hard see any differences between the two models, as $K_{a c t}$ is close to unity for typical values of $\phi$ and $\delta$.

\subsection{Final system of conservation laws}

Many observations (Melosh 1986; Dent et al. 1998; Keller, Ito \& Nishimura 1998) suggest that the downslope velocity profile with depth is rather blunt, and shear is confined to a very thin layer. To order $\varepsilon^{1+\gamma}$ the downslope velocity is assumed to be independent of depth, implying

$$
u^{b}=\bar{u}+O\left(\varepsilon^{1+\gamma}\right), \quad u^{s}=\bar{u}+O\left(\varepsilon^{1+\gamma}\right), \quad \overline{u^{2}}=\bar{u} \bar{u}+O\left(\varepsilon^{1+\gamma}\right) .
$$

Using these relations together with the expressions for the longitudinal pressure (2.32), or (2.34), the final system of conservation laws for the avalanche motion with accumulation and deposition is

$$
\begin{gathered}
\partial_{t} h+\partial_{x}(h u)=d^{s}-d^{b}, \\
\partial_{t}(h u)+\partial_{x}\left(h u^{2}\right)+\varepsilon \partial_{x}\left(K \cos \zeta h^{2} / 2\right)=h D-\varepsilon h \cos \zeta \partial_{x} b+u\left(d^{s}-d^{b}\right),
\end{gathered}
$$

where, for simplicity, the averaging bar is now dropped on the downslope velocity component. The driving force $D$ is given by (2.28) and the earth pressure coefficient $K$ is either defined by (2.31), or is equal to 1 in the inviscid case. Given a slope inclination angle $\zeta$, basal friction angle $\delta$, internal friction angle $\phi$, the basal topography $b$, the surface accumulation rate $d^{s}$ and the basal deposition rate $d^{b}$, this forms a closed system of equations for the avalanche depth $h$ and the depth-averaged downslope velocity $u$. 


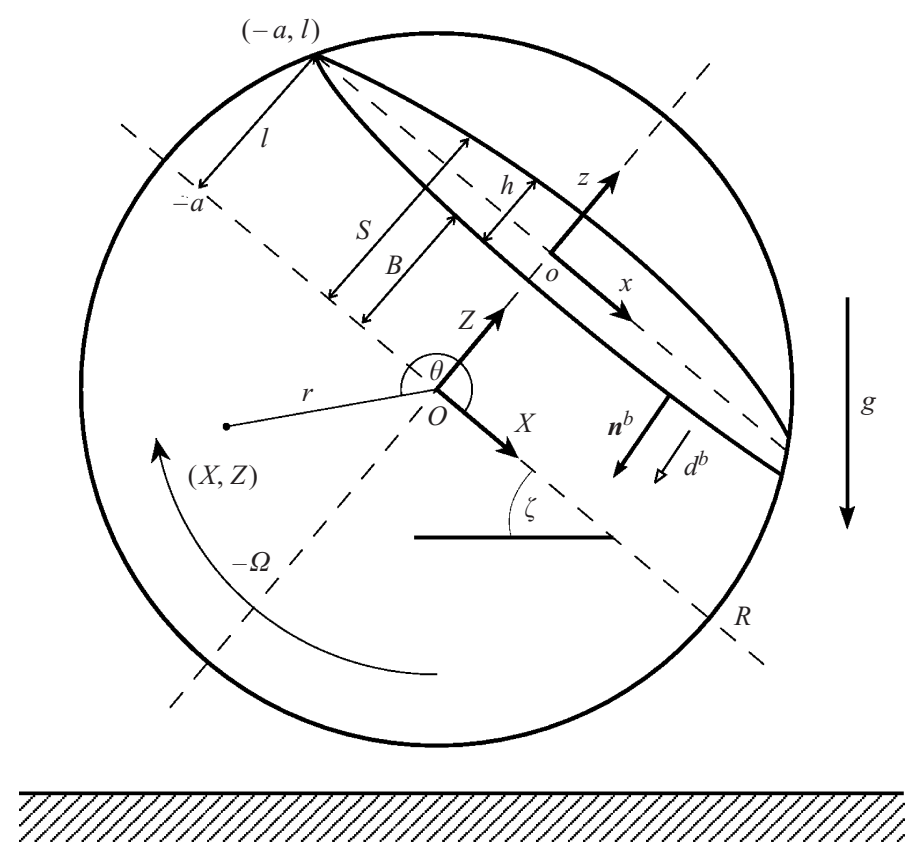

FIGURE 2. A sketch of a rotating drum of radius $R$ partially filled with granular material. A Cartesian coordinate system $o x z$ is defined with the $x$-axis inclined at an angle $\zeta$ to the horizontal, so that it lies approximately parallel to the avalanche free surface when the material is at failure. The origin $o$ lies close to the avalanching region. A second coordinate system $O X Z$ is defined such that its axes are parallel to those of $o x z$, but the origin $O$ lies on the axis of rotation at the centre of the drum. The components in the two coordinate systems are related by $X=x$ and $Z=l+z$, where $l$ is defined as the fill level of the drum. The free surface of the avalanche lies at $Z=S(X, t)$ and the interface between the avalanching and solid granular material lies at $Z=B(X, t)$. The avalanche thickness $h(X, t)$ is measured parallel to the $Z$-axis. In steady flow the top of the avalanche is taken to lie at $(-a, l)$ and mass balance implies $h=0$ when $u \neq 0$ at the drum wall. In unsteady flow, however, a shock wave of finite thickness may be generated if $u=0$ at the wall. Strong mass transfer takes place between the avalanche and the solid, the deposition rate $d^{b}$ is the equivalent volume of granular material that is eroded from the avalanche base per unit area per unit time. The angular velocity $\Omega$ is positive for rotation in the counter-clockwise sense. The above configuration is for clockwise rotation and the angular velocity is denoted by $-\Omega$.

\section{A theory for granular flow in rotating drums}

\subsection{Coordinate system}

It is convenient to use two Cartesian coordinate systems $o x z$ and $O X Z$ to reflect the different geometries in the avalanching and solid body regions. The avalanche coordinate system $o x z$ was defined in the previous section. The angle of inclination $\zeta$ is chosen so that the downslope $x$-axis lies approximately parallel to the free surface when the material is at failure. The $z$-axis is normal to it and points upward. The $O X Z$ coordinate system is defined so that its axes are parallel to those in $o x z$, but the origin $O$ is shifted so that it lies on the axis of revolution, as illustrated in figure 2. The two coordinate systems are therefore related by

$$
Z=l+z, \quad X=x,
$$

where the constant $l$ is defined as the fill level of the drum. This definition ensures that the origin $o$ of the coordinate system oxz lies at most a distance $H$ from the avalanche body, where $H$ is a typical avalanche thickness. It follows from (3.1) that the free 
surface height, $S(X, t)$, and singular surface height, $B(X, t)$, in the $O X Z$ system are related to their counterparts, $s(x, t)$ and $b(x, t)$ in the $o x z$ system by

$$
S=l+s, \quad B=l+b .
$$

The rotating drum is taken to have a radius $R$. Typical length scales in the solid region will therefore be of order $R$, in both the $X$ - and $Z$-directions. The avalanche is also of length $R$ in the $x$-direction, but because the avalanche is shallow, its thickness is only of order $H$. As $H \ll R$ the shallowness approximation holds for avalanches in rotating drums and the theory of the preceding section can be directly applied. The motion of the avalanche can therefore be computed by solving the system of equations (2.36)-(2.37) together with the earth pressure coefficient (2.31), once appropriate interfacial conditions have been prescribed.

\subsection{Governing equations in the solid rotating granular material}

Velocity and density fields within the solid material will be denoted with the superscript - , to avoid confusion with these fields in the avalanche. It is assumed that the solid granular material is a rigid body, of constant uniform density $\rho^{-}$, which rotates with angular velocity $\Omega(t)$ about the axis of revolution $O$. At the boundary between the granular material and the drum slip may occur, and the angular velocity of the drum, $\Omega^{d}(t)$, is in general, not equal to $\Omega$. The velocity field is therefore simply

$$
\boldsymbol{u}^{-}=\Omega r \hat{\boldsymbol{\theta}}
$$

where $r$ is the distance from the axis of revolution $O$ and $\hat{\boldsymbol{\theta}}$ is the azimuthal unit vector. It follows that the downslope and normal velocity components in the solid are

$$
u^{-}=-\Omega Z, \quad w^{-}=\Omega X .
$$

At the non-material singular interface, $F^{b}$, with the avalanching granular material, the body is subject to a kinematic boundary condition

$$
F^{b}(\boldsymbol{x}, t)=0: \quad \partial_{t} F^{b}+\boldsymbol{v}^{b-} \cdot \nabla F^{b}=0,
$$

where $\boldsymbol{v}^{b-}$ is the velocity of the interface. The superscript notation $b+$ and $b-$ is introduced to differentiate between variables that are evaluated on the avalanching side (upper side) and solid body side (lower side) of the singular interface, $F^{b}$, respectively.

\subsection{Interfacial conditions and scalings}

At the interface between the avalanche and the rotating granular material there are discontinuities in the velocity and density fields. At such a singular surface the mass jump condition (e.g. Chadwick 1976) is

$$
\llbracket \rho\left(\boldsymbol{u} \cdot \boldsymbol{n}^{b}-v_{n}^{b}\right) \rrbracket=0,
$$

where the jump bracket, $\llbracket f \rrbracket=f^{b+}-f^{b-}$, is the difference between the value on the upper and lower sides of the singular surface. This provides a coupling condition between the velocities in the avalanche and the solid. If the typical angular velocity magnitude of the rotating granular material is $\Omega^{*}$, then the velocities in the solid body are of magnitude $\Omega^{*} R$. Assuming that density changes are relatively small, a balance between the normal velocity components in (3.6) implies that $\Omega^{*} R=\varepsilon(g R)^{1 / 2}$.

The scalings (2.7) hold true for the avalanche in the rotating drum. New scalings are now introduced for the fill height, the free and singular surface variations, and 
the variables in the solid rotating body:

$$
\left.\begin{array}{l}
(X, Z, l, B, S)=R(\tilde{X}, \tilde{Z}, \tilde{l}, \tilde{B}, \tilde{S}), \\
(\Omega)=\varepsilon(g / R)^{1 / 2}(\tilde{\Omega}), \\
\left(u^{-}, w^{-}, v_{n}^{b-}\right)=\varepsilon(R g)^{1 / 2}\left(\tilde{u}^{-}, \tilde{w}^{-}, \tilde{v}_{n}^{b-}\right),
\end{array}\right\}
$$

where the tildes are again used to indicate non-dimensional variables. In the avalanche the downslope and normal length scales are scaled differently to reflect the shallowness of the geometry, whilst in the solid body the coordinates are scaled using the same length scales. Using (3.1) these differential scalings imply that the non-dimensional coordinates are related by

$$
Z=l+\varepsilon z, \quad X=x,
$$

where for simplicity the tildes are dropped. Henceforth all variables are nondimensional unless stated otherwise. It follows that the free surface height and the interface height are

$$
S=l+\varepsilon S, \quad B=l+\varepsilon b .
$$

The velocity components in the solid body are

$$
u^{-}=-\Omega Z, \quad w^{-}=\Omega X,
$$

and, in particular, the velocity components on the lower side of the singular surface are

in coordinates $o x z$.

$$
u^{b-}=-\Omega(l+\varepsilon b), \quad w^{b-}=\Omega x,
$$

Let us suppose that the normal deposition rate of material of density $\rho^{-}$into the solid body per unit area per unit time is $d^{b-}$, then the velocity of the basal interface $\boldsymbol{v}^{b-}=\boldsymbol{u}^{b-}-d^{b-} \boldsymbol{n}^{b}$. The normal interface speeds $\boldsymbol{v}^{b+} \cdot \boldsymbol{n}^{b}=\boldsymbol{v}^{b-} \cdot \boldsymbol{n}^{b}=v_{n}^{b}$ to prevent internal void space opening up between the avalanche and the solid body. Thus, if the avalanche density on the upper side of the singular surface is $\rho^{+}$the mass jump condition (3.6) implies that

$$
d^{b+}=\left(\rho^{-} / \rho^{+}\right) d^{b-},
$$

where the ratio of the densities is close to unity. The velocities on the lower (solid body) side of the singular surface are prescribed by the nature of the rigid rotation (3.11). It follows from the kinematic condition (3.5) that

$$
d^{b-}=-\varepsilon \Omega l \partial_{x} b-\Omega x+\partial_{t} b+O\left(\varepsilon^{2}\right),
$$

which together with (3.12) provides an approximation for the deposition rate $d^{b+}$ to order $\varepsilon^{1+\gamma}$.

\subsection{Governing equations in the avalanche region}

The fluid-like region in the rotating drum can be modelled as a granular avalanche with erosion and deposition at its basal interface. The theory of the previous section is therefore appropriate and the coordinate system $o x z$ and all the variables, scalings and results are adopted here. In addition the avalanche is assumed to have constant density $\rho^{+}=\rho_{0}$ and the surface accumulation, $d^{s}$, defined in the previous section is taken equal to zero. The conservation laws therefore reduce to

$$
\begin{gathered}
\partial_{t} h+\partial_{x}(h u)=-d^{b+}, \\
\partial_{t}(h u)+\partial_{x}\left(h u^{2}\right)+\varepsilon \partial_{x}\left(K \cos \zeta h^{2} / 2\right)=h D-\varepsilon h \cos \zeta \partial_{x} b-u d^{b+},
\end{gathered}
$$


to $O\left(\varepsilon^{1+\gamma}\right)$, where the deposition rate $d^{b+}$ is given by (3.12) and (3.13), and the earth pressure coefficient is defined in (2.31). In the rotating drum experiments there is a downslope velocity component in the solid region. This implies that the relative velocity $u^{r}=u-\varepsilon \Omega l+O\left(\varepsilon^{2}\right)$, and the source term

$$
D=\cos \zeta(\tan \zeta-\operatorname{sgn}(u-\varepsilon \Omega l) \tan \delta) .
$$

\section{Steady-state solutions}

\subsection{An exact solution for steady flow}

Steady-state solutions to the rotating drum theory are now investigated. It is therefore assumed that solid granular material rotates with constant angular velocity, $\Omega_{0}$, and that all derivatives with respect to time are zero, $\partial_{t}()=0$. The basal friction angle $\delta(x)$ is assumed to be equal to a constant value $\delta_{0}$. It follows that if the coordinate system $o x z$ is chosen such that its angle of inclination $\zeta=\delta_{0}$, then the net driving force, $D$, (defined in (3.16)) is equal to zero, provided that the magnitude of the avalanche velocity is greater than $\left|\varepsilon \Omega_{0} l\right|$. For classical smooth solutions the conservative form of the momentum balance (3.15) can be simplified with the help of the mass balance (3.14) to yield

$$
\begin{gathered}
\partial_{x}(h u)=\left(\rho^{-} / \rho^{+}\right)\left(\varepsilon \Omega_{0} l \partial_{x} b+\Omega_{0} x\right), \\
h u \partial_{x} u+\varepsilon \partial_{x}\left(K \cos \zeta h^{2} / 2\right)=-\varepsilon h \cos \zeta \partial_{x} b .
\end{gathered}
$$

Usually the avalanche equations (4.1)-(4.2) are solved for the avalanche thickness, $h$, and velocity, $u$, given suitable initial and boundary conditions and basal topography, $b$.

Here a special class of solutions is considered in which the downslope avalanche velocity is constant,

$$
u=u_{0}
$$

and equations (4.1)-(4.2) are solved for the avalanche thickness, $h$, and the basal topography, $b$, given suitable boundary conditions. Since $\partial_{x} u=0$ the earth pressure coefficient is equal to a constant value $K_{0}$ throughout the avalanche. For the inviscid model $K_{0}=1$, in the Savage \& Hutter (1989) theory $K_{0}=K_{a c t}$, and in the regularized model of Tai \& Gray (1998) $K_{0}=2 \sec ^{2} \phi-1$. For constant $K=K_{0}$ the governing equations reduce to

$$
\begin{gathered}
-\lambda \partial_{x} h=\varepsilon l \partial_{x} b+x, \\
K_{0} \partial_{x} h=-\partial_{x} b,
\end{gathered}
$$

where the order-unity constant

$$
\lambda=-\frac{\rho^{+} u_{0}}{\rho^{-} \Omega_{0}} .
$$

For solutions with positive avalanche thicknesses we shall show that $\lambda$ is positive. It may therefore be assumed, without loss of generality, that the downslope avalanche velocity is positive, $u_{0}>0$, and hence that the angular velocity is negative, $\Omega_{0}<0$.

The uppermost point of intersection between the avalanche free surface and the drum wall defines the top of the avalanche and lies at $(-a, l)$ in the $O X Z$ system, where $l$ is the fill level and $a=\left(R^{2}-l^{2}\right)^{1 / 2}$. Integrating equations (4.4) and (4.5) subject to the boundary conditions $h=0$ and $b=0$ at $x=-a$ implies

$$
\begin{gathered}
-\lambda h=\varepsilon l b-\left(a^{2}-x^{2}\right) / 2, \\
K_{0} h=-b .
\end{gathered}
$$



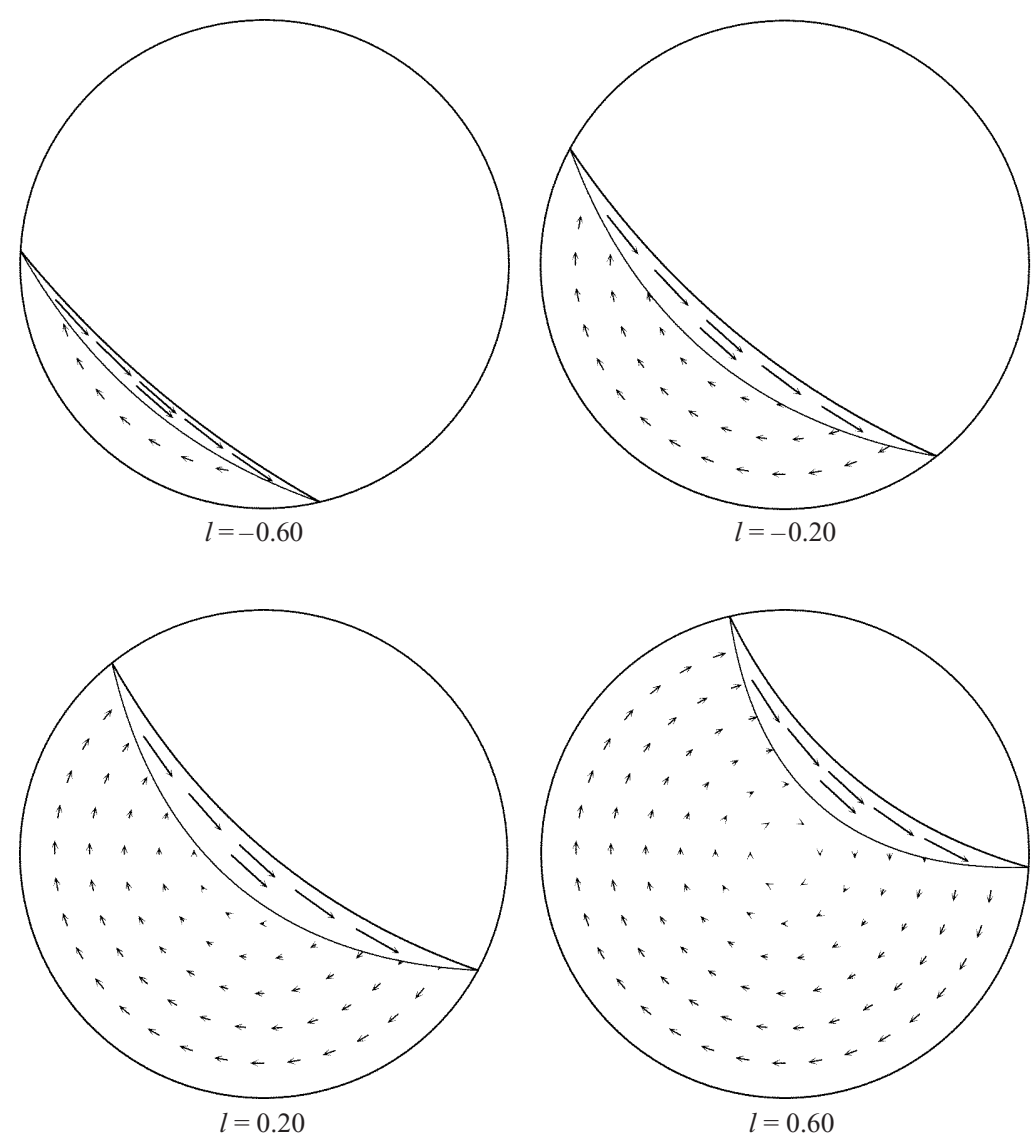

FIGURE 3. The steady-state solution for the flow of granular material in a partially filled rotating drum is illustrated for four differing fill heights. In each case $R=1, \rho^{-} / \rho^{+}=1.05, K_{0}=2.0, \lambda=3$ and $\delta_{0}=40^{\circ}$. The position of the avalanche free surface and the interface between the avalanching and solid regions are illustrated, as well as velocity vectors to show the relative speed and direction of the flow.

It follows that the avalanche thickness and basal topography are

$$
\begin{gathered}
h=h_{0}\left(a^{2}-x^{2}\right) / a^{2}, \\
b=-K_{0} h_{0}\left(a^{2}-x^{2}\right) / a^{2},
\end{gathered}
$$

where the constant

$$
h_{0}=\frac{a^{2}}{2\left(\lambda-\varepsilon l K_{0}\right)}
$$

is the avalanche thickness at $x=0$. For positive order-unity values of $\lambda$ the constant $h_{0}$ and hence the avalanche thickness $h$ are positive, confirming our original assumption. Given free parameters $\lambda, \delta_{0}, l$ and $R$, and using one of the constitutive models to determine $K_{0}$ the system of equations can be used to predict the avalanche thickness $h$ and the position of the free surface $b$.

The explicit solution is plotted in figure 3 , assuming that $K_{0}>1$, to give the avalanche a characteristic crescent shape with a concave free surface for each of the four fill levels. In the inviscid model $\left(K_{0}=1\right)$ the solution is similar except the free surface is straight. If $K_{0}<1$ then the free surface would be convex. In the experiments presented here the free surface is slightly concave. 
The basal topography gradients imply that a point mass would be accelerated in the region $x<0$ and decelerated in the region $x>0$. The avalanche thickness gradients decelerate the avalanche in the region $x<0$ and accelerate it in the region $x>0$. An exact balance between these competing effects ensures that there is no net acceleration and the downslope avalanche velocity is constant along its length.

In the unstretched coordinate system, when $\varepsilon$ is set equal to unity the nondimensional parameter $\lambda$ becomes large. Using the scalings (2.7) and (3.7) it is easily shown that $\lambda=\left(\rho^{+} u_{0}^{*}\right) /\left(-\rho^{-} \Omega_{0}^{*} R\right)$, where the starred variables $u_{0}^{*}$ and $\Omega_{0}^{*}$ are typical dimensional magnitudes of the downslope and angular velocities, respectively. The downslope avalanche velocity, $u_{0}^{*}$, is therefore much larger than the maximum speed in the rotating granular material, $R \Omega_{0}^{*}$. The parameter $\lambda$ is a measure of the ratio of the maximum avalanche to solid body speeds, and typically lies in the range $10 \leqslant \lambda \leqslant 30$. The solutions in figure 3 are presented for the case $\lambda=3$, i.e. downslope avalanche velocities are three times larger than the solid body velocity close to the drum wall. This value of $\lambda$ allows the velocity vectors to be plotted on the same diagrams and illustrates the effect of the $\varepsilon$ terms in (4.11). For positive fill levels the coupling with the solid body velocity field implies that the avalanche is thicker than for negative values of $l$. In addition the curvature of the basal topography is larger for increasing fill levels, which has also been observed in the experiments. As $\lambda$ is increased the avalanche thickness and curvature decrease and the velocity increases.

\subsection{Particle paths}

The particle paths within the avalanching flow can be determined by integrating the differential equations

$$
\frac{\mathrm{d} x}{\mathrm{~d} t}=u_{0}, \quad \frac{\mathrm{d} z}{\mathrm{~d} t}=w,
$$

subject to the initial conditions that $x=x_{0}$ and $z=z_{0}$ at $t=0$. The derivative $\mathrm{d} / \mathrm{d} t$ is the rate of change as observed when moving with a fixed particle. It follows that

$$
x=u_{0} t+x_{0},
$$

since the downslope velocity is constant. The normal velocity $w$ can be determined from the incompressibility relation (2.8) subject to the interfacial condition (3.6). Since the downslope velocity is constant incompressibility implies that $\partial_{z} w=0$. Integrating this with respect to $z$ gives

$$
w=w^{b+},
$$

where the normal velocity at the base of the avalanche

$$
w^{b+}=u_{0} \partial_{x} b+\left(\rho^{-} / \rho^{+}\right) \Omega_{0}\left(\varepsilon l \partial_{x} b+x\right)+O\left(\varepsilon^{2}\right) .
$$

Substituting (4.14) and (4.15) into the differential equation for the normal velocity component (4.12) and using (4.13) to make the change of variables $t=\left(x-x_{0}\right) / u_{0}$ implies that

$$
\lambda \frac{\mathrm{d} z}{\mathrm{~d} x}=\lambda \partial_{x} b-\left(\varepsilon l \partial_{x} b+x\right)+O\left(\varepsilon^{2}\right) .
$$

Integrating with respect to $x$ implies that the particle height as a function of position is

$$
z=z_{0}+b-(\varepsilon l / \lambda) b-x^{2} /(2 \lambda)+O\left(\varepsilon^{2}\right),
$$

where $z_{0}$ is the initial height of the particle.

To aid the process of tracking particles it is convenient to introduce a new parameter 


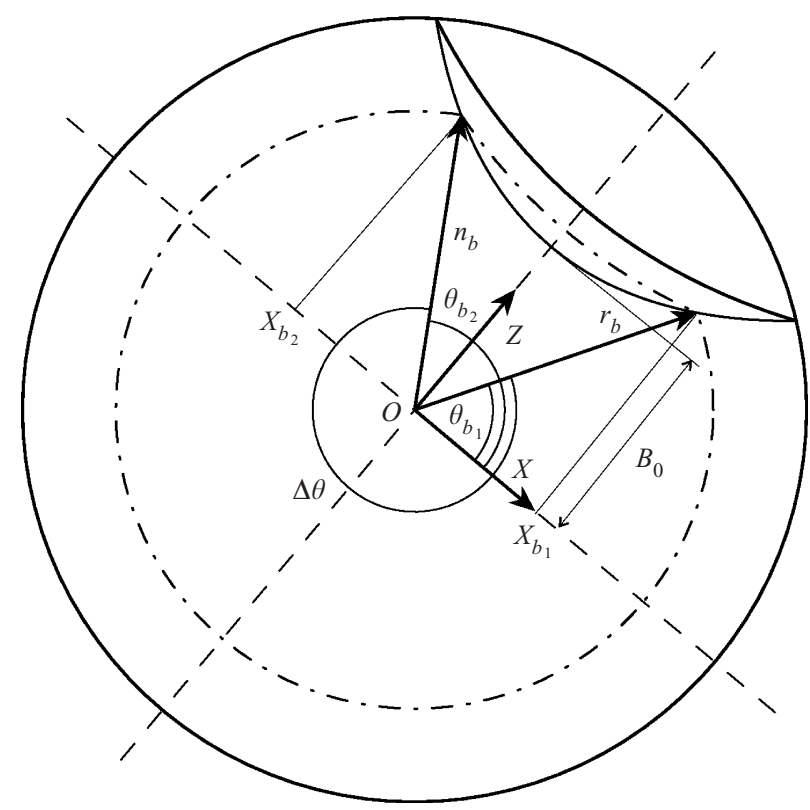

FIGURE 4. A sketch showing a closed particle path (dot-dash line) for steady granular flow inside a partially filled drum. At position $\left[r_{b}, \theta_{b_{1}}\right]$ particles leave the avalanching region and rotate rigidly until they reach position $\left[r_{b}, \theta_{b_{2}}\right]$, where they re-enter the avalanche and are transported to $\left[r_{b}, \theta_{b_{1}}\right]$ again to complete the circuit. The angle between $\theta_{b_{1}}$ and $\theta_{b_{2}}$ measured through the region of solid rotation is $\Delta \theta$. The base of the avalanche at $X=0$ lies at $B_{0}$. If $B_{0}$ is greater than zero a solid core develops in the centre of the drum and the particles in this region never enter into the avalanche.

$\alpha$ to label the particle paths that pass through the avalanching domain. The maximum height of the avalanche is attained at $x=0$ and therefore all the avalanche particle paths cross the $z$-axis. Let $\alpha$ be the relative height of the avalanche particle path to the maximum avalanche height $h_{0}$ as it crosses the $z$-axis. It follows that $\alpha$ is linear in $z$ and equal to zero at the base of the avalanche and unity at the free surface. The value of $\alpha$ uniquely labels the particle paths that pass through the avalanche.

It will now be shown that the avalanching particle paths form closed curves that pass through the fluid- and solid-like regions. Using the definition of $\alpha$ above it follows from (4.17) that the particle height

$$
z=b+h_{0} \alpha-(\varepsilon l / \lambda)\left(b-b_{0}\right)-x^{2} /(2 \lambda)+O\left(\varepsilon^{2}\right),
$$

where $b_{0}=-K_{0} h_{0}$ is the position of the solid/avalanche interface at $x=0$. Equation (4.18) can be simplified by substituting for the basal topography, from (4.10) and (4.11), in the third term on the right-hand side to give

$$
z=b+h_{0} \alpha-h_{0}(x / a)^{2}+O\left(\varepsilon^{2}\right) .
$$

A particle crosses the interface between the avalanche and the solid rotating granular material when its height is equal to the height of the local basal topography, i.e. when $z=b$. In this case (4.19) becomes a quadratic equation for the intersection positions and has two real roots

$$
x_{b_{1}}=a \sqrt{\alpha}=x_{b}, \quad x_{b_{2}}=-a \sqrt{\alpha}=-x_{b},
$$

where $x_{b}=a \sqrt{\alpha}$. These points lie an equal downslope distance on either side of the $z$-axis. This is illustrated in figure 4 . The associated normal components of the 
intersection points are

$$
z_{b_{1}}=b\left(x_{b_{1}}\right), \quad z_{b_{2}}=b\left(x_{b_{2}}\right),
$$

respectively, where $b$ is given by (3.9) and (4.10). As the intersection points lie an equal downslope distance on either side of the origin and since the basal interface, $b$, is an even function, it follows that the intersection points have the same normal components $z_{b_{1}}=z_{b_{2}}\left(=z_{b}\right.$ say). Assuming that the velocity $u_{0}$ is positive, a particle will cross from the solid to the avalanche at $\left\{x_{b_{2}}, z_{b}\right\}$ and pass back from the avalanche to the solid at $\left\{x_{b_{1}}, z_{b}\right\}$, where the curly brackets $\{$,$\} denote components in the oxz system. In the$ $O X Z$ system these points correspond to $\left(X_{b_{2}}, Z_{b}\right)$ and $\left(X_{b_{1}}, Z_{b}\right)$, respectively, where $X_{b_{1}}=x_{b_{1}}, X_{b_{2}}=x_{b_{2}}$ and $Z_{b}=l+\varepsilon z_{b}$. Both of these positions lie an equal distance,

$$
r_{b}=\sqrt{X_{b}^{2}+Z_{b}^{2}},
$$

from the axis of rotation at the centre of the drum.

The particles in the solid granular material are in rigid rotation about the origin and their paths are given by solving

$$
\frac{\mathrm{d} X^{-}}{\mathrm{d} t}=-\varepsilon \Omega_{0} Z^{-}, \quad \frac{\mathrm{d} Z^{-}}{\mathrm{d} t}=\varepsilon \Omega_{0} X^{-}
$$

subject to the initial conditions that $X^{-}=X_{0}^{-}$and $Z^{-}=Z_{0}^{-}$at $t=0$. It is simple to show that the particles move in circular arcs that are parameterized by

$$
X^{-}=r \cos \theta, \quad Z^{-}=r \sin \theta,
$$

where the azimuthal angle

$$
\theta=\varepsilon \Omega_{0} t+\theta_{0}
$$

and $\theta_{0}=\tan \left(Z_{0}^{-} / X_{0}^{-}\right)$.

An avalanche particle path intersects the basal interface at positions $\left(X_{b_{1}}, Z_{b}\right)$ and at $\left(X_{b_{2}}, Z_{b}\right)$. In polar coordinates these points have positions $\left[r_{b}, \theta_{b_{1}}\right]$ and $\left[r_{b}, \theta_{b_{2}}\right]$, respectively, where the angles

$$
\begin{aligned}
& \theta_{b_{1}}=\cos ^{-1}\left(X_{b_{1}} / r_{b}\right), \\
& \theta_{b_{2}}=\cos ^{-1}\left(X_{b_{2}} / r_{b}\right) .
\end{aligned}
$$

It follows that after a particle has crossed from the avalanche to the solid at $\left[r_{b}, \theta_{b_{1}}\right]$ it then describes a circular arc until it reaches $\left[r_{b}, \theta_{b_{2}}\right]$, where it re-enters the avalanche. As both the starting and re-entry positions lie on the same circular arc it follows that the particle paths in the rotating drum form closed curves that extend through both the avalanching and solid body regions. Each of these closed curves is identified by the relative height $\alpha$ as it crosses the $x$-axis in the avalanche. The solutions are illustrated in figure 5 for four different fill heights.

A particularly interesting feature of the flow is that if the height of the basal topography lies above the axis of rotation at $x=0$, a solid central core develops in which the particles never enter into the avalanche. That is, if the height of the basal interface at $x=0$

$$
\left.\begin{array}{ll}
B_{0}>0: & \text { a solid core develops, } \\
B_{0} \leqslant 0: & \text { no solid core, }
\end{array}\right\}
$$

where $B_{0}=l+\varepsilon b_{0}$. For positive $B_{0}$ the radius of the central core is $B_{0}=l-\varepsilon K_{0} h_{0}$. Thus, as the fill level increases, the size of the central core becomes progressively larger as shown in the lower two panels of figure 5. This agrees well with laboratory 

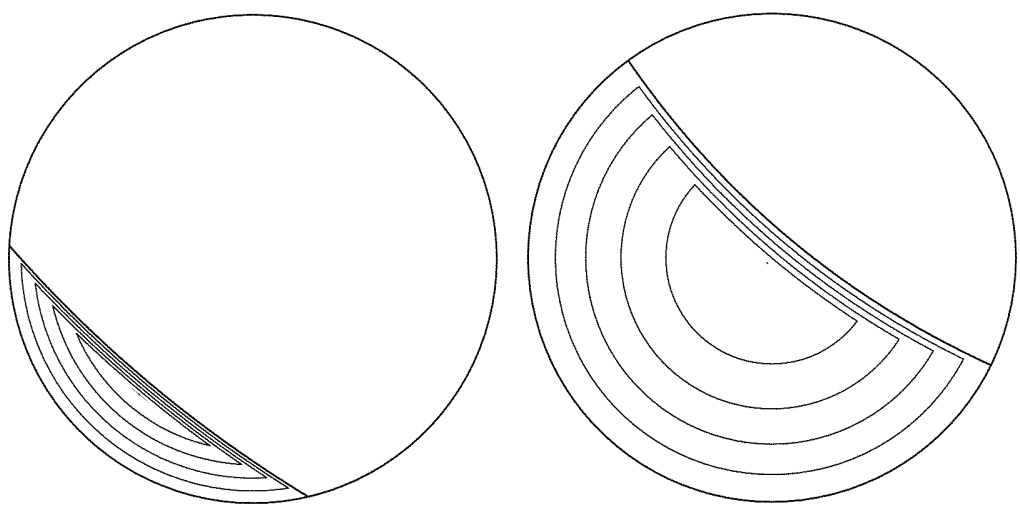

$B_{0}=-0.72, l=-0.60$

$B_{0}=-0.03, l=0.24$

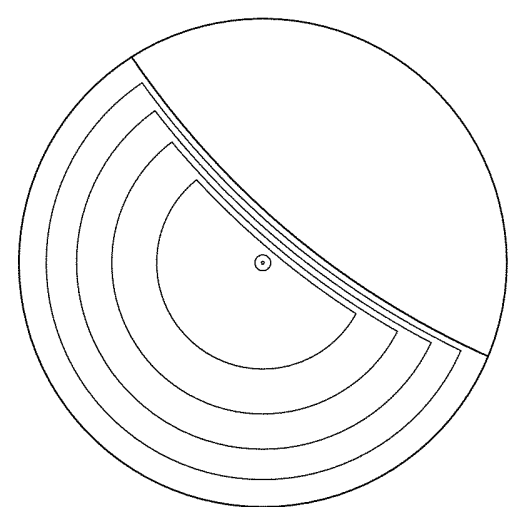

$B_{0}=0.03, l=0.30$

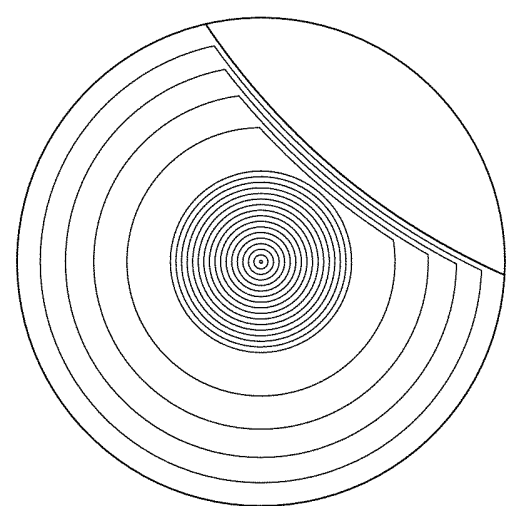

$B_{0}=0.37, l=0.60$

FIGURE 5. A series of particle paths is shown for four differing fill heights. In each case $R=1$, $\lambda=4, \rho^{-} / \rho^{+}=1.05, K_{0}=2.0$ and $\delta_{0}=40^{\circ}$. For $B_{0} \leqslant 0$ (in the upper two panels) the steady-state particle paths all form closed curves that pass through both the avalanching and rotating regions of the granular material. In the case $B_{0}>0$ (in the lower two panels) there is an additional central solid core in which the particles remain entirely in the rigid rotating region of the granular material.

experiments. Figure 6 shows four long time exposures of a drum partially filled with black and white poppy seeds. In the upper two panels the height of the basal interface $B_{0}<0$, which implies that all the particle paths intersect with the avalanching domain close to the free surface. In the lower two panels the height of the basal interface $B_{0}>0$, so a solid core develops in which the particles are always in solid rotation.

\subsection{Circuit times}

The time a particle takes to complete a circuit around the drum is extremely sensitive to the fill level of the drum, $l$, and the particle path, $\alpha$. This has very important consequences for the mixing of granular material, which will be shown experimentally in the next section. A particle entering the avalanche at position $\left\{-x_{b}, z_{b}\right\}$ takes a time

$$
t_{a}=2 x_{b} / u_{0}
$$

to reach the other end of the avalanche at $\left\{x_{b}, z_{b}\right\}$. Once the particle leaves the avalanche it travels along a circular arc in the solid region from $\left[r_{b}, \theta_{b_{1}}\right]$ to $\left[r_{b}, \theta_{b_{2}}\right]$ 

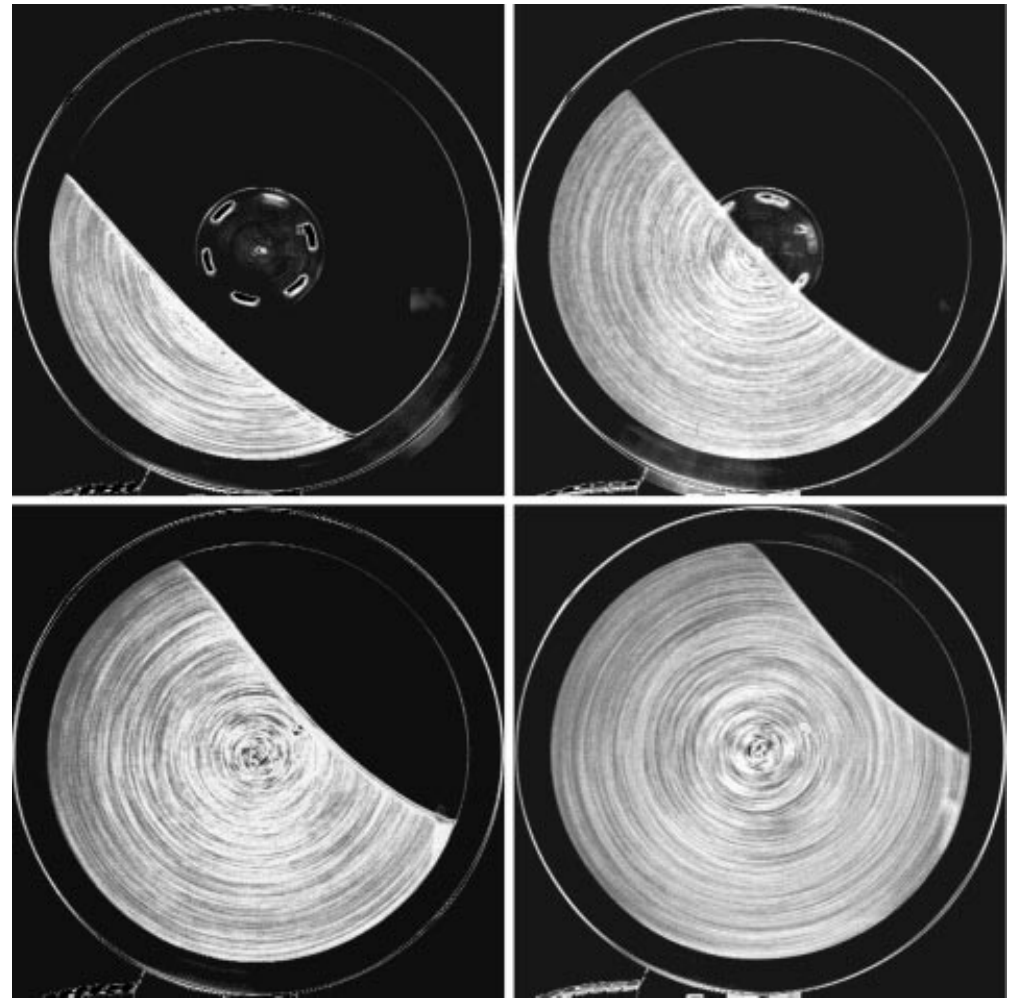

FIGURE 6. Long time exposures showing the particle paths in a rotating drum partially filled with black and white poppy seeds. In the solid rotating region the particles describe circular arcs. For $B_{0} \leqslant 0$ in the upper two panels all the particle paths pass through the avalanche near the free surface. For $B_{0}>0$ (lower two panels) some of the particles never intersect with the free surface and perform closed circuits entirely within the solid body region. For higher rotation speeds and large drum sizes the free surface may become asymmetric.

taking a time

$$
t_{s}=\Delta \theta /\left(-\varepsilon \Omega_{0}\right)
$$

where

$$
\Delta \theta=\operatorname{ang}\left(\theta_{b 1}, \theta_{b 2}\right)
$$

is the angle between the intersection points measured through the solid region. The angle lies in the range $0 \leqslant \Delta \theta \leqslant 2 \pi$. The factor $\varepsilon^{-1}$ in (4.30) implies that the time spent in the solid region is large compared to the time spent in the avalanche, i.e. $t_{s} \gg t_{a}$. Note that the solutions in this and subsequent sections are all plotted with the aspect ratio $1: 1$, which is achieved by setting $\varepsilon=1$ in the avalanche flow solutions to unstretch the coordinates. For simplicity, the angular velocity is chosen so that the drum performs one complete revolution in a single non-dimensional time unit, i.e. $\Omega_{0}=-2 \pi$. It follows that the particles travel through the avalanche in a time, $t_{a} \ll 1$.

The total time $t_{t}$ taken for a particle to perform one complete circuit of the drum is the sum of the time spent in the avalanche $t_{a}$ and the time spent in solid rotation $t_{s}$

$$
t_{t}=t_{a}+t_{s}
$$




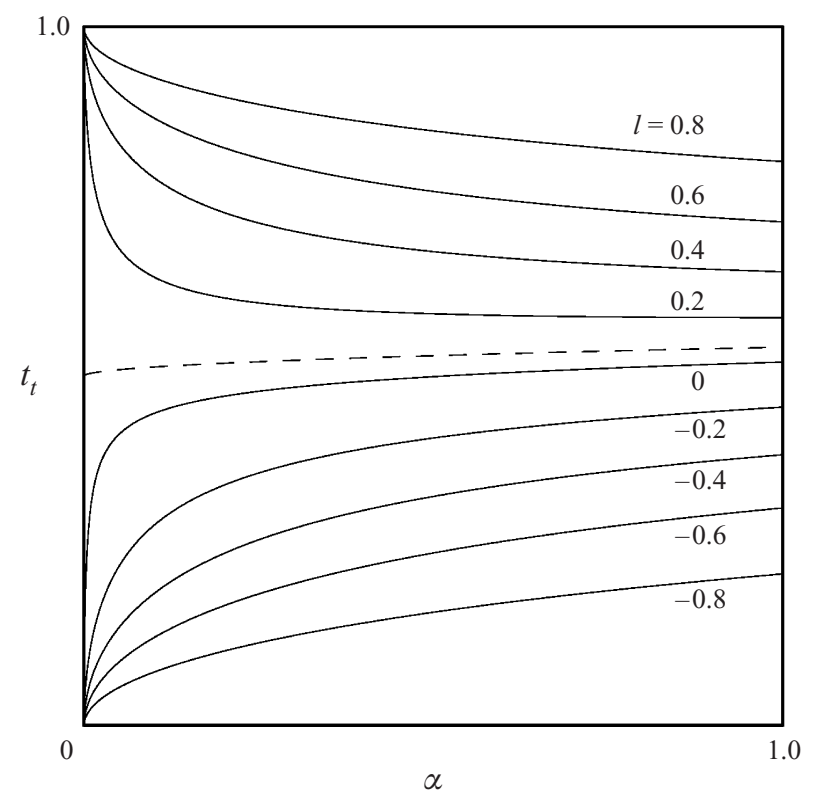

FIGURE 7. The total time for a particle to complete one circuit around the drum, $t_{t}$, is plotted as a function of the particle path $\alpha$ for a series of fill levels, $l$. In each case $R=1, \lambda=15, \rho^{-} / \rho^{+}=1.05$, $K_{0}=2.0, \delta_{0}=40^{\circ}$ and $\Omega_{0}=-2 \pi$. Recall, that when $\alpha=1$ the particles move around paths adjacent to the drum wall and the free surface of the avalanche, whilst when $\alpha=0$ the particles move around the circumference of the solid core or lie at the singular point $\left(0, B_{0}\right)$. The dashed line marks the transition between flows with a central solid core, above the line, and those without, below the line.

This is plotted in figure 7 as a function of the particle path $\alpha$ for a series of fill levels, $l$. In general, when a solid core develops $\left(B_{0}>0\right)$ grains that start on higher particle paths, $\alpha$, perform complete circuits of the drum faster than those on lower ones. Close to the interface $B_{0}=0$ interface curvature effects can cause the minimum circuit time to occur internally, but this is a weak effect. At the point where the base of the avalanche $\left(0, B_{0}\right)$ coincides with the axis of revolution, i.e. $B_{0}=0$, there is a very sudden transition, which is indicated by the dashed line in figure 7 . For $B_{0} \leqslant 0$ the solid core disappears and grains that start on higher particle paths, $\alpha$, take longer to perform a circuit than those on lower paths. At the point $\left(0, B_{0}\right)$ there is singular behaviour since it takes zero time to perform a circuit, which leads to very intense mixing. These features will be explained with the help of the experiments of the next section.

\section{Steady mixing of granular material}

\subsection{Experimental configuration}

The difference between the total time, $t_{t}$, to complete a circuit on different particle paths, $\alpha$, leads to an apparent shearing or mixing of the granular material. Metcalfe et al. (1995) designed a simple experiment to make this deformation and mixing visible. In their experiments a divider was placed along the drum diameter and equal amounts of differently coloured granular material were poured on either side. The divider was then removed and the drum closed, so that the initial configuration was similar to that shown in figure 8 . As the drum rotated the initially straight interface between the coloured regions was deformed making the mixing visible. 


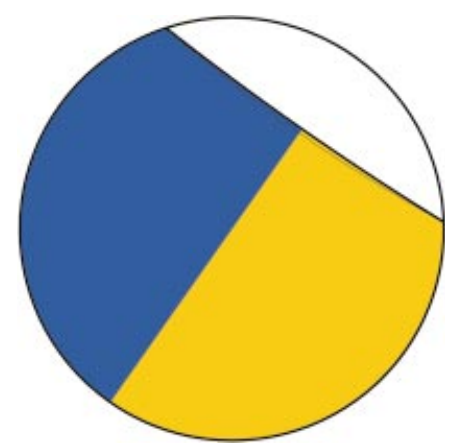

FIGURE 8. The granular material is initially divided into two regions of equal mass with one half shaded yellow and the other blue. All the particles are of the same size, surface roughness and density and differ only in their colour. The drum is rotated with constant angular velocity and the two regions mix during steady flow. The avalanche takes place in a thin layer close to the free surface. The interface between the avalanche and solid body region below it is shown by the thin black line.

In the experiments performed by Metcalfe et al. (1995) and McCarthy et al. (1996) the drum was rotated so slowly that they were in the intermittent flow regime, in which discrete avalanches were observed that stopped completely before the next one began. Here experiments have been performed to investigate the mixing in the steady continuous flow regime, which develops for constant rotation periods in the range $100-10 \mathrm{~s}$ per revolution. Initially the drum was filled with equal masses of yellow and blue coloured quartz sand (as in figure 8) of the same density, surface roughness and grain size distribution $(0.35-0.65 \mathrm{~mm})$. The drum, of diameter $25 \mathrm{~cm}$ and width $3 \mathrm{~mm}$, was then slowly rotated at constant speed. The narrow width was not an essential feature the experiment, since three-dimensional effects are weak and the motion is largely planar.

\subsection{Fill level $l=0.6$}

A series of 24 photographs of the experimental results for a fill level $l=0.6$ is shown in figure 9 . The photographs begin at the top left corner after $1 / 8$ of a revolution and are illustrated at $1 / 8$ of a revolution intervals ending at the bottom left after three complete revolutions. Time increases from left to right and from top to bottom. The central solid core is immediately apparent as no deformation or mixing occurs in this region and the interface remains straight.

Outside the central core the particle paths transport the grains through both the solid- and fluid-like regions. The graph in figure 7 implies that grains on higher particle paths, $\alpha$, should perform circuits faster than those on lower paths. This can clearly be seen in the experiments. Consider the upper portion of the interface between the yellow and blue sand after $3 / 8$ of a revolution (third image from the left on the top row) in figure 9. It is still straight as it has not been deformed during the rigid rotation in the solid body. After $4 / 8$ of a revolution (right image on the top row) the interface has passed through the avalanche and been deformed. The reason for this is not because the particles themselves travel any faster, but simply because of where they cross the singular surface, $b$. The angle between the entry and exit points measured through the solid body, $\Delta \theta$, which was defined in (4.31), is smaller for higher particle paths, $\alpha$, than for lower ones. It follows that a grain simply spends less time in slow solid rotation, and gets reabsorbed into the solid body ahead of particles of lower particle paths, $\alpha$. The interface between the yellow and blue sand is 

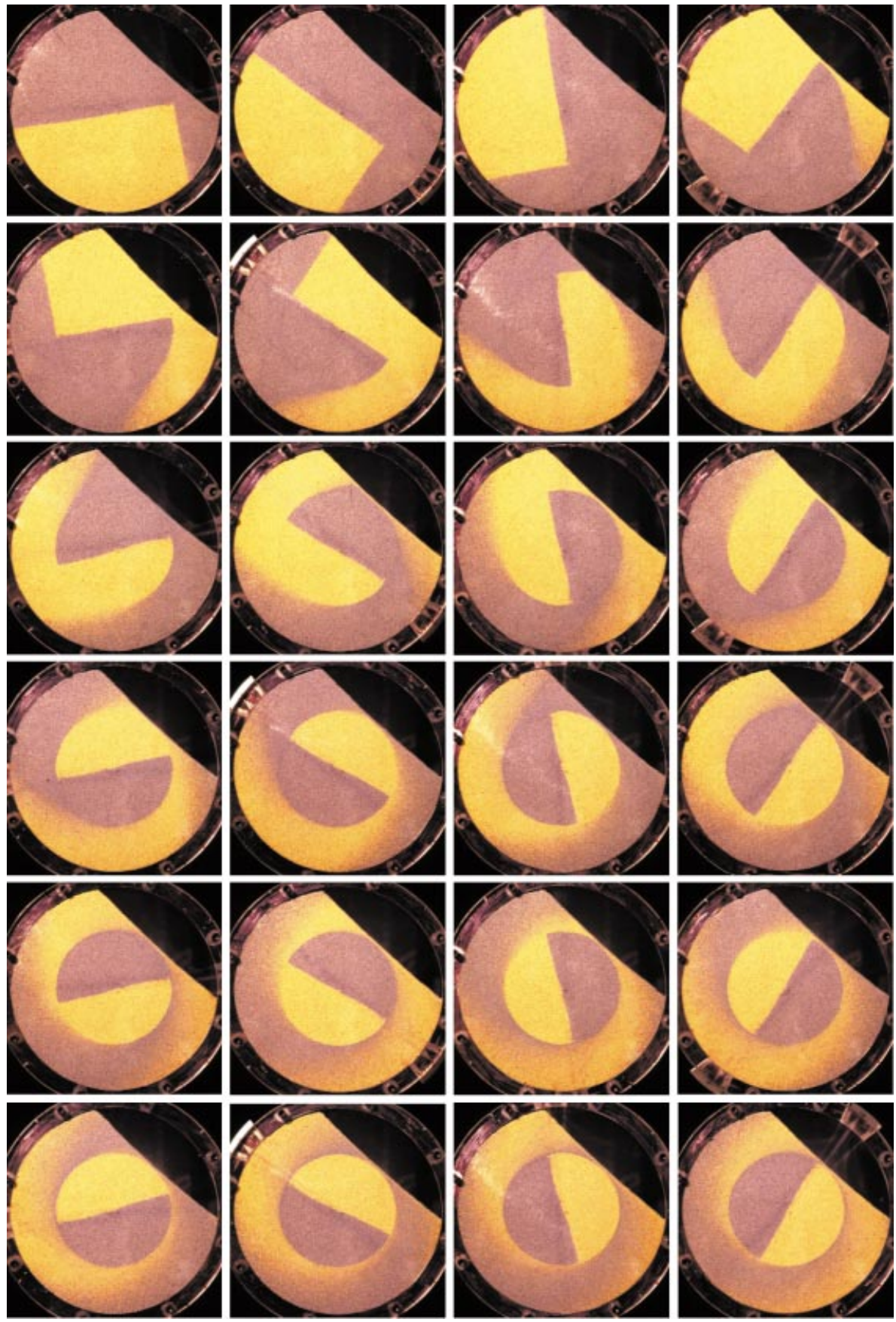

FIGURE 9. Photographs showing the mixing of blue and yellow sand during steady continuous flow in a drum rotating at $100 \mathrm{~s}$ per revolution. The drum is of diameter $25 \mathrm{~cm}$ and width $3 \mathrm{~mm}$. The sands are identical except for the colour and have a particle size distribution in the range $0.35-0.65 \mathrm{~mm}$. The fill level $l=0.6$. The sequence of photographs begins at top left, after $1 / 8$ of a revolution, and ends at bottom right after three revolutions. The photographs are taken after each $1 / 8$ of a revolution. 
therefore deformed so that it appears to 'radiate' away from the axis of rotation in a clockwise sense.

The same portion of the interface starts to enter the avalanche again after $9 / 8$ revolutions (left-hand image on the third row in figure 9). As the interface has already been deformed it takes longer for all the particles on the interface to enter and exit the avalanche. Particles that lie just above the solid core are the slowest, and do not exit until 12/8 revolutions have occurred (right-hand image on the third row in figure 9). The interface is stretched again and the deformation is compounded. This demonstrates that the differences between the circuit times, on different particle paths, accumulate linearly after each rotation.

The steady flow solution (4.9)-(4.11) together with the particle tracking and circuit time information can easily be used to model continuous mixing in a partially filled rotating drum. A series of marker points are used to track the position of the interface between the yellow and blue sand. In the theory there is no transport or diffusion across this interface and the marker points simply move around the particle paths. If a marker point is placed on a known particle path $\alpha$ it stays on this path for all time.

Given the initial position of a marker point the problem is to find its position at any given time $T$. Each circuit around path $\alpha$ takes a known time $t_{t}$, which is defined in (4.29)-(4.32). This makes tracking the particle easy as the total number of complete rotations around the circuit $\alpha$ is simply

$$
n=\operatorname{int}\left(T / t_{t}\right),
$$

where 'int' is the integer part. The problem therefore reduces to finding the position of a particle in the finite time interval $0 \leqslant \tau<t_{t}(\alpha)$, where $\tau$ is the truncated time

$$
\tau=T-n t_{t} .
$$

Since the path and the velocity along the path are known it is a simple matter to compute the position of the particle at the truncated time $\tau$.

Figure 10 shows the steady flow solution for the interface position at a series of 24 non-dimensional times between $1 / 8$ and 3 non-dimensional time units. Recalling that the drum completes one revolution in unit time, these times intervals correspond to $1 / 8$ of a drum revolution and the images are directly comparable to those of the experiment in figure 9. In the simulations the granular material is assumed to dilate by $5 \%$ as it fluidizes, the avalanche mass flux to rigid rotational mass flux $\lambda=15$ and the slope inclination is assumed to be $35^{\circ}$. These parameters were chosen to approximate the actual conditions in the experiments. Particles at the base of the avalanche, $\left(0, B_{0}\right)$, lie on the path $\alpha=0$ and take unit time to rotate with the solid core. The fastest circuits of the drum are performed by particles on the path $\alpha=1$, which lies along the free surface and the drum wall. The position and size of the interface and central core are in good agreement with experiment. The exact steady flow solution predicts the exact position of a sharp interface between the yellow and blue sand. In the experiments random collisions between the particles as they travel through the avalanche introduce some fluctuations that diffuse the flow. Each time the interface passes through the avalanche it is therefore progressively diffused. The effects of self-diffusion of the particles, in the avalanche, is not included in the theory.

$$
\text { 5.3. Fill level } l=0.2
$$

Laboratory experiments have also been performed for a fill level of $l=0.2$. A series of 16 photographs of the experimental results is shown in figure 11 at $1 / 8$ of a 

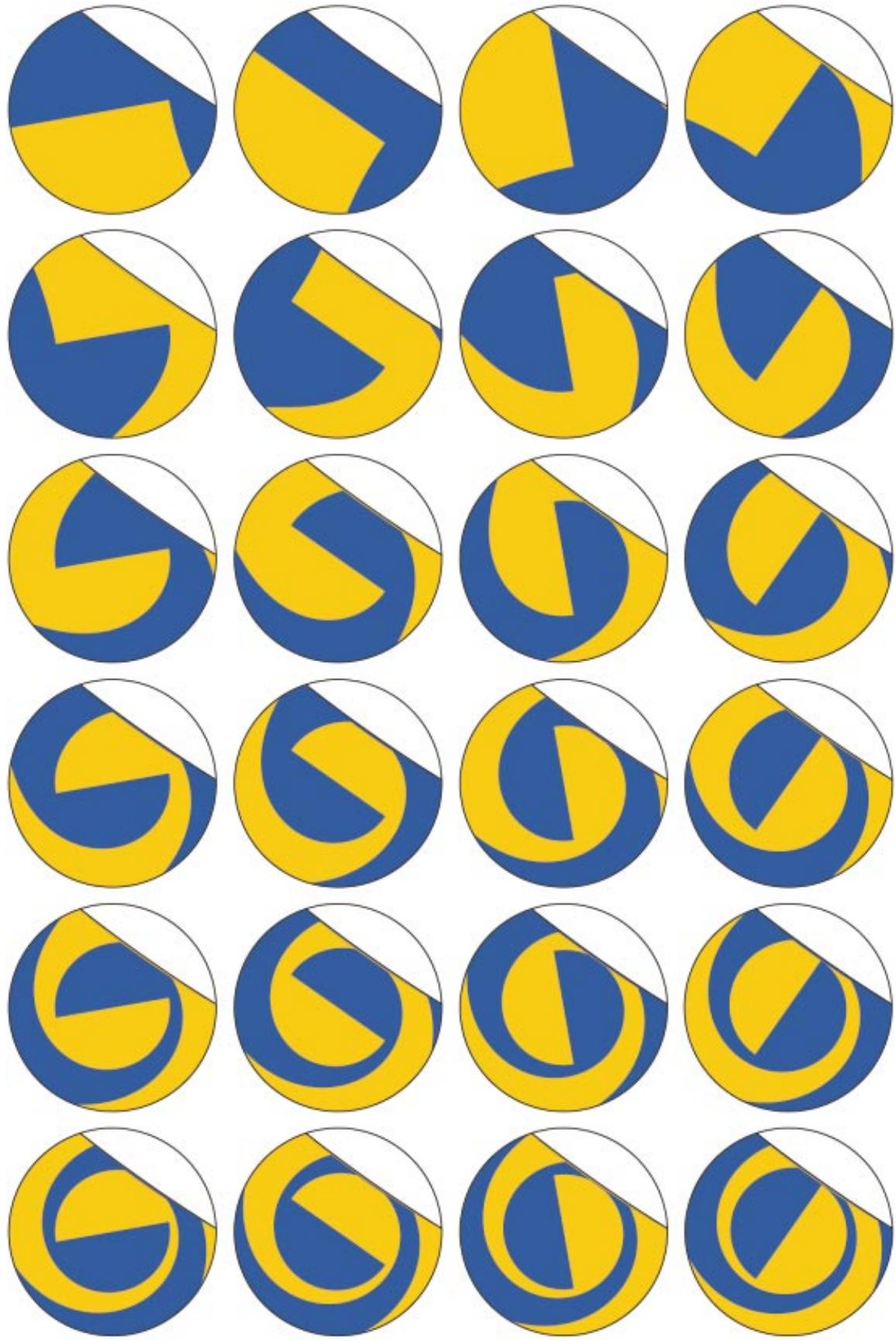

FIGURE 10. The mixing of a monodisperse granular material in a drum rotating with constant angular velocity. The fill level $l=0.6$ and the grains are initially divided so that yellow particles lie on the right-hand side and blue particles on the left-hand side of the drum. The steady flow solution is illustrated for a sequence of 24 equal time steps ranging from $1 / 8$ to 3 non-dimensional time units, top left to bottom right. The standard parameters $R=1$, $\lambda=15, K_{0}=2, \rho^{-} / \rho^{+}=1.05, \delta_{0}=35^{\circ}$ and $\Omega_{0}=-2 \pi$ are used. One revolution of the drum equates to one non-dimensional time unit. A computer animation of the solution is available at http://www.ma.man.ac.uk/ ngray/Drums/drums.html/. 


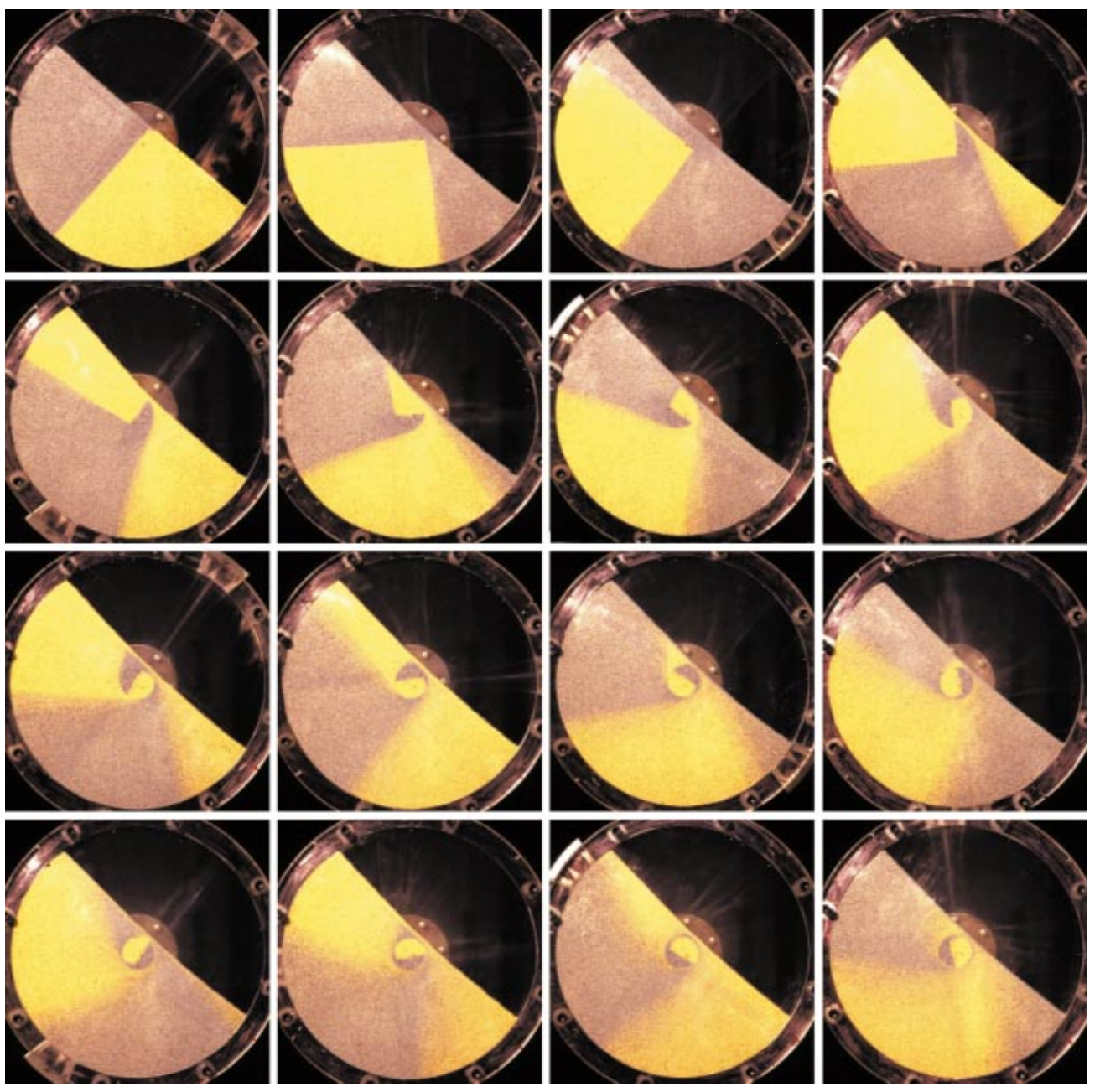

FIGURE 11. As figure 9 but for fill level $l=0.2$. The sequence of photographs begins in the top left and ends in the bottom right after $15 / 8$ revolutions. The photographs are taken after each $1 / 8$ of a revolution.

revolution intervals. A central core also develops here, but it is much smaller than in the previous experiment. Most of the stretching of the interface between the yellow and blue regions occurs in a region close to the solid core. As figure 7 predicts, there is a large difference in the circuit times between particles in the solid core and particles that lie on paths $\alpha<0.2$. Within this region particles on paths with larger $\alpha$ values perform circuits much faster than particles in the solid core. The interface is therefore stretched in this region, so that it 'radiates' out in a clockwise sense from the axis of revolution. In the region $\alpha \geqslant 0.2$ the circuit times are almost all the same and there is very little stretching of the interface. This explains why the interface between the yellow and blue regions remains approximately straight outside the neighbourhood of the central core.

The larger the fill height of the drum the more time it takes for a particle on path $\alpha>0$ to perform a complete circuit. It follows that the interface between the yellow and blue regions wraps itself around the central core much faster in the case $l=0.2$ 

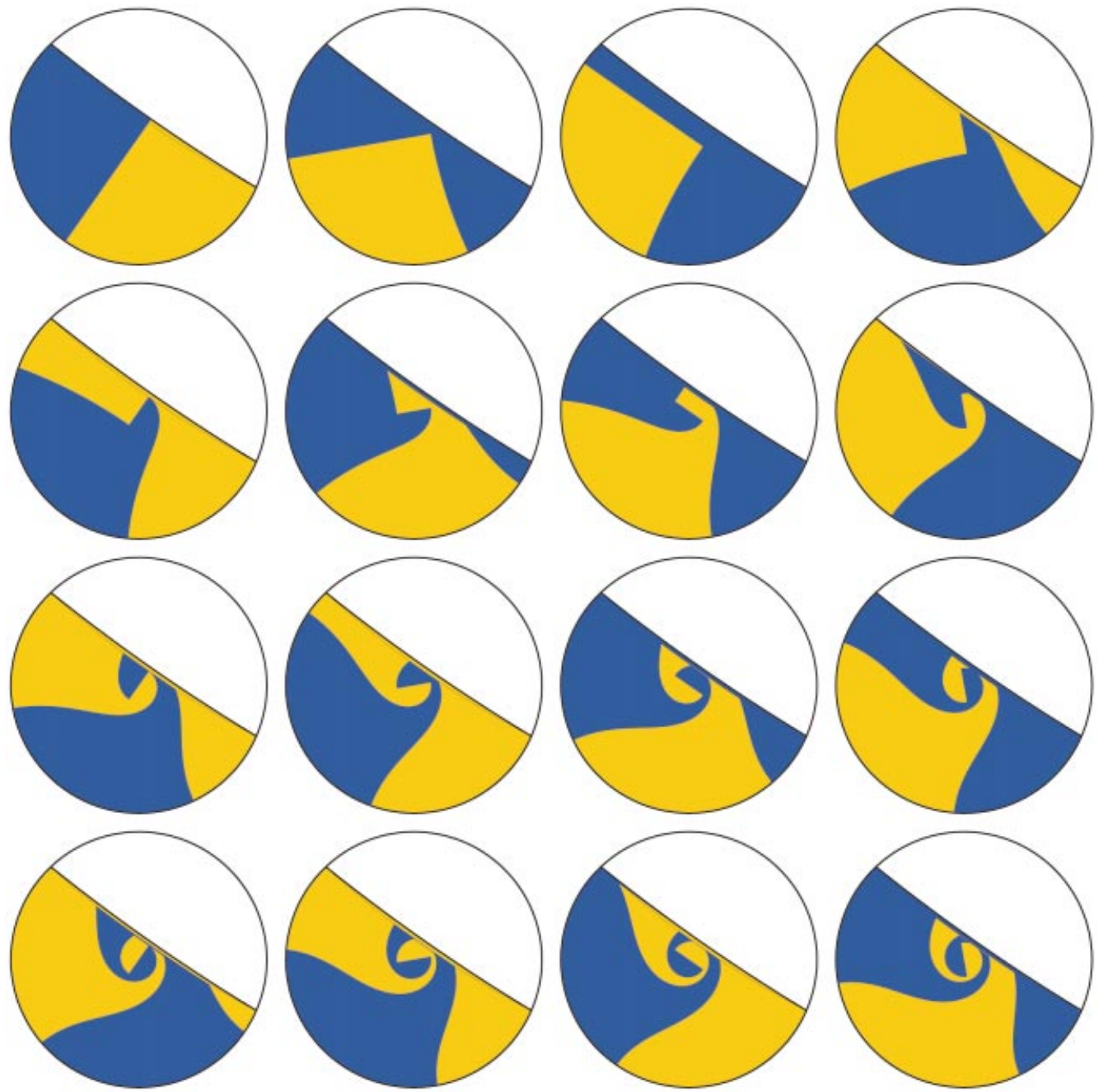

Figure 12. As figure 10 but for fill level $l=0.2$. The steady flow solution is illustrated for a sequence of 16 equal time steps starting at 0 and ending at $15 / 8$ non-dimensional time units.

than in the case of $l=0.6$. This is easily seen by comparing the photographs of the drum for fill level $l=0.6$ (right-hand image, second row of figure 9) and fill level $l=0.2$ (left-hand image, third row figure 11) after one complete revolution of the drum.

Figure 12 shows the corresponding steady flow solution at intervals of $1 / 8$ of a non-dimensional time unit. The interface remains almost straight outside the neighbourhood of the central core, indicating that the circuit times of the particle paths are almost constant. Whilst close to the central core the stretching is concentrated in a narrow region, which wraps itself around the core more rapidly than for larger fill heights in accordance with the results in figure 7. Particles on the path $\alpha=1$ perform more circuits, and travel through the avalanche more often, than for the same path at a fill level $l=0.6$. It follows that the experimental interface is slightly more diffuse than in the previous experiment after the same number of drum revolutions. 

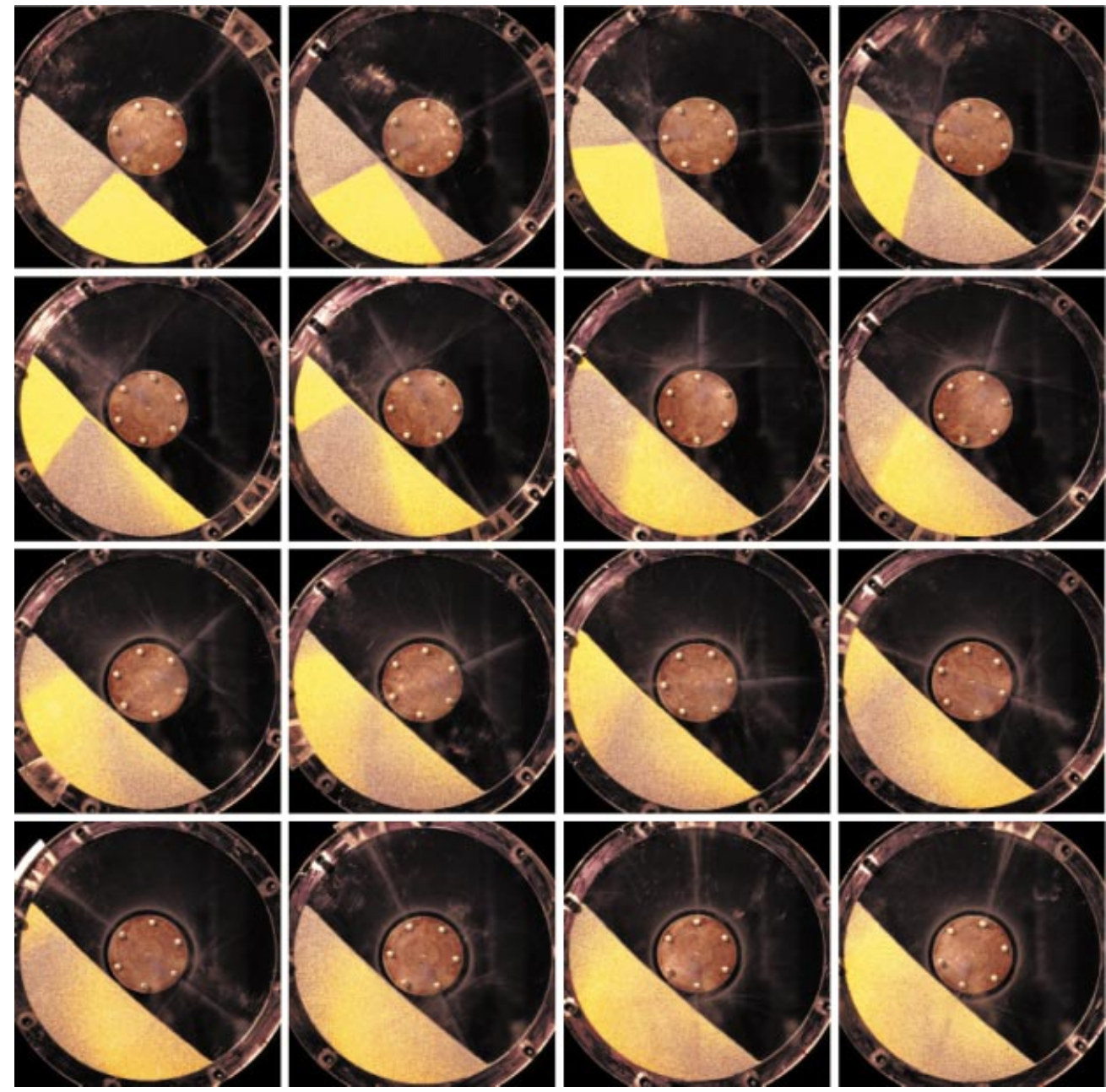

Figure 13. As figure 9 but for fill level $l=-0.3$. The sequence of photographs begins in the top left and ends in the bottom right after 15/16 of a revolution. The photographs are taken after each $1 / 16$ of a revolution.

\subsection{Fill level $l=-0.3$}

When the fill level lies below the centre of the drum the character of the explicit solutions and the experimental results change dramatically. A series of 16 photographs of the experiment are shown in figure 13 at $1 / 16$ of a revolution intervals. In direct contrast to the case $B_{0}>0$, grains on higher particle paths, $\alpha$, move round the drum slower those on lower ones. Again the reason for this is simply the position at which they cross the singular surface, $b$. The angle between the entry and exit points measured through the solid body, $\Delta \theta$, is larger for higher particle paths, $\alpha$, than for lower ones, when the interface lies below the axis of revolution. These particles must therefore spend more time in slow solid body rotation. It follows that the interface is deformed so that it 'radiates' away from the axis of revolution in an anti-clockwise sense. This can be clearly seen in the first eight images in figure 13 .

Figure 14 illustrates the steady explicit solution at time increments corresponding to those in the experiment. The interface position is in very good agreement for the 

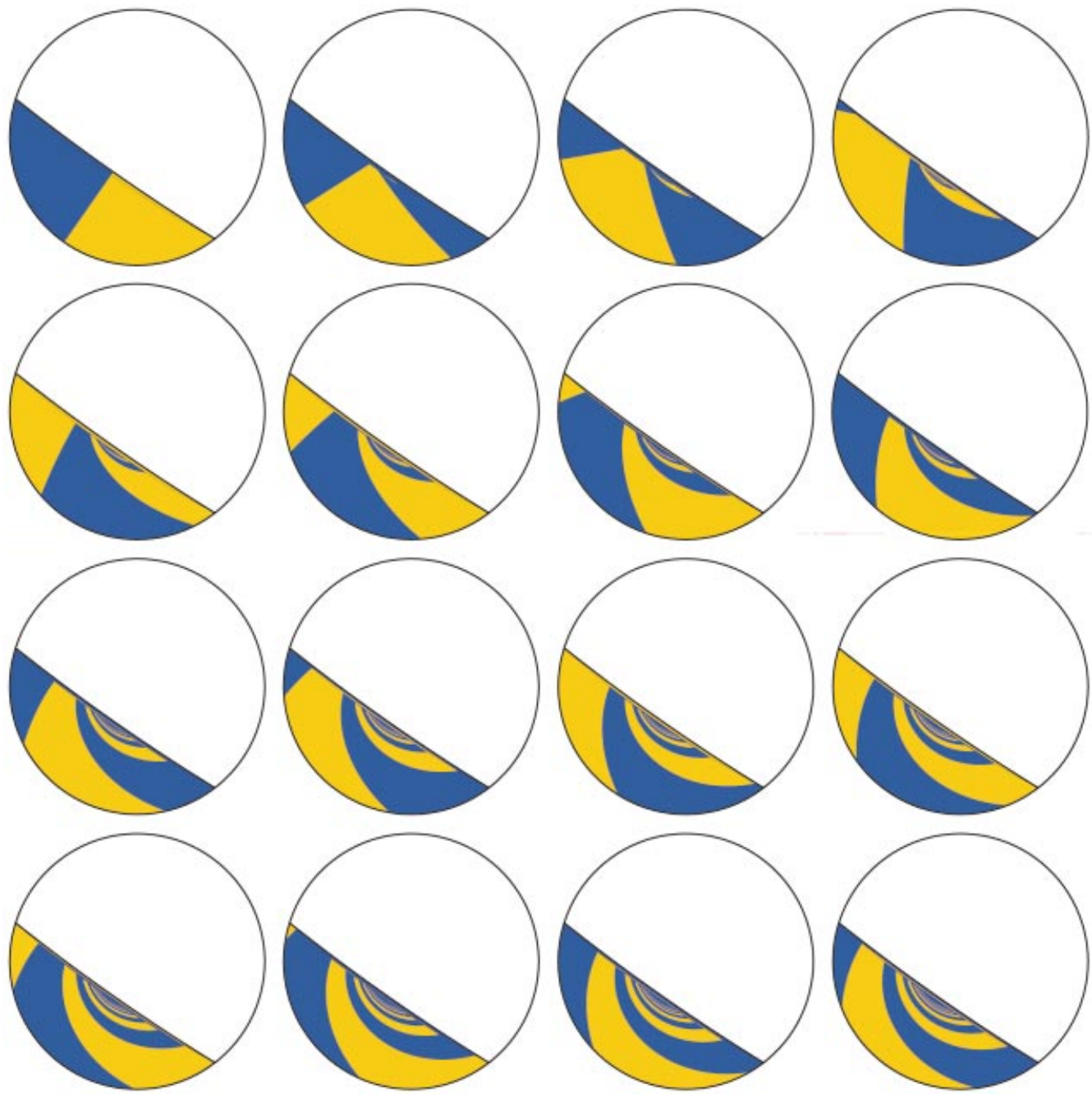

Figure 14. As figure 10 but for fill level $l=-0.3$. The steady flow solution is illustrated for a sequence of 16 equal time steps starting at 0 and ending at 15/16 of a non-dimensional time unit, top left to bottom right.

early time intervals. It 'radiates' away from the axis of revolution in an anti-clockwise sense, as in the experiments, indicating that the particles closer to the drum wall and the free surface lag behind those in the centre of the flow. After about half a drum revolution the interface has been deformed so much that the explicit solution develops extremely fine-scale features close to $\left(0, B_{0}\right)$, which are well below the grain scale. The cause of this is that in the limit as $\alpha \rightarrow 0$, the time $t_{t}$ for particles to perform circuits tends to zero, as shown by the graph in figure 7. The interface therefore wraps itself around the singular point very quickly, passing through the avalanche many times and undergoing a large amount of diffusion. Since the exact steady-state solution is an idealization that does not take grain size effects or diffusion into account, it does not reproduce the experiments well close to the singular point $\left(0, B_{0}\right)$. 


\section{Discussion}

A theoretical framework has been derived for the flow of granular materials in partially filled slowly rotating drums. The assumption that the fluid-like avalanche can be coupled to the solid rotating region by a non-material singular surface has been shown to be a good one. The dominant physical processes are the dynamics of the avalanche, mass transfer across the singular surface and the slow rotation of the solid phase. In steady flow it is sufficient to consider the solid phase as a rigid rotating body that is coupled to the avalanche phase by a mass jump condition. A simple assumption then allows the complete flow field to be derived exactly. A particularly interesting feature of the theory is that the particle paths form closed curves that pass through both the avalanching and solid body regions. This is a good approximation to what actually occurs in the experiments, although self-diffusive phenomena are not modelled. When the base of the avalanche lies above the axis of revolution, $B_{0}>0$, a solid core develops in the centre of the drum. A dramatic transition in the flow behaviour occurs when the base of the avalanche coincides with, or lies below, the axis of revolution $\left(B_{0} \leqslant 0\right)$. The solid core disappears and the point $\left(0, B_{0}\right)$ is singular causing rapid mixing. These effects are demonstrated during the steady mixing of two differently coloured granular materials.

This research was supported by the Deutsche Forschungsgemeinschaft through the SFB 298 project 'Deformation und Versagen bei metallischen und granularen Strukturen'. The exact solution was derived whilst on a NSC-DAAD exchange programme at the National Taiwan University, Taipei. This paper is dedicated to Professor Kolumban Hutter on the occasion of his 60th birthday.

\section{REFERENCES}

Chadwick, P. 1976 An Introduction to Continuum Mechanics. George, Allen \& Unwin.

Dent, J. D., Burrel, K. J., Schmidt, D. S., Louge, M. Y., Adams, E. E. \& Jazbutis, T. G. 1998 Density, velocity and friction measurements in a dry-snow avalanche. Annal. Glaciol. 26, 247-252.

GenNes, P. G. DE 1999 Dynamique superficielle d'un matériau granulaire. Preprint.

Gray, J. M. N. T. \& HutTer, K. 1997 Pattern formation in granular avalanches. Contin. Mech. Thermodyn. 9(6), 341-345.

Gray, J. M. N. T. \& Hutter, K. 1998 Physik granularer Lawinen. Physikalische Blätter 54(1), $37-43$.

Gray, J. M. N. T. \& TAI Y. C. 1998 Particle size segregation, granular shocks and stratification patterns. In Physics of Dry Granular Media (ed. H. J. Herrmann et al.), pp. 697-702. NATO ASI series, Kluwer.

Gray, J. M. N. T., Wieland, M. \& Hutter K. 1999 Free surface flow of cohesionless granular avalanches over complex basal topography. Proc. R. Soc. Lond. A 455, 1841-1874.

Greve, R. \& Hutter, K. 1993 Motion of a granular avalanche in a convex and concave curved chute: experiments and theoretical predictions. Phil. Trans. R. Soc. Lond. A 342, 573-600.

Greve, R., Koch, T. \& Hutter, K. 1994 Unconfined flow of granular avalanches along a partly curved surface. Part I: Theory Proc. R. Soc. Lond. A 445, 399-413.

Hutter, K. 1996 Avalanche dynamics. In Hydrology of Disasters (ed. V. P. Singh), Water Sciences and Technology, vol. 24, pp. 317-394. Kluwer.

Hutter, K. \& Greve, R. 1993 Two-dimensional similarity solutions for finite mass granular avalanches with Coulomb and viscous-type frictional resistance. J. Glaciol. 39, 357-372.

HutTer, K. \& Koch, T. 1991 Motion of a granular avalanche in an exponentially curved chute: experiments and theoretical predictions. Phil. Trans. R. Soc. Lond. A 334, 93-138.

Hutter, K., Koch, T., Plüss, C. \& Savage, S. B. 1995. The dynamics of avalanches of granular materials from initiation to runout. Acta Mech. 109, 127-165. 
Hutter, K. \& Nohguchi, Y. 1990 Similarity solutions for a Voellmy model of snow avalanches with finite mass. Acta Mech. 82, 99-127.

Hutter, K., Siegel, M., Savage, S. B. \& Nohguchi, Y. 1993 Two-dimensional spreading of a granular avalanche down an inclined plane. Part I Theory. Acta Mech. 100, 37-68.

JiAng, G. \& TADMOR, E. 1997 Non-oscillatory central schemes for multidimensional hyperbolic conservation laws. SIAM J. Sci. Comput. 19, 1892-1917.

Keller, S., Ito, Y. \& Nishimura, K. 1998 Measurements of the vertical velocity distribution in ping pong ball avalanches. Annal. Glaciol. 26, 247-252.

Koch, T., Greve, R. \& Hutter, K. 1994 Unconfined flow of granular avalanches along a partly curved surface, Part II: Experiments and numerical computations. Proc. R. Soc. Lond. A 445, 415-435.

McCarthy, J. J, Wolf, J. E., Shinbrot, T. \& Metcalfe, G. 1996 Mixing of granular materials in slowly rotated containers. AIChE J. 42, 3351-3363.

Metcalfe, G, Shinbrot, T, McCarthy, J. J. \& Ottino, J. M. 1995 Avalanche mixing of granular solids. Nature 374, 39-41.

Melosh, J. 1986 The physics of very large landslides. Acta Mech. 64, 89-99.

Nessyahu, H. \& TADMOR, E. 1990 Non-oscillatory central differencing for hyperbolic conservation laws. J. Comput. Phys. 87, 408-463.

RAJCHENBACH, J. 1990 Flow in powders: From discrete avalanches to continuous regime. Phys. Rev. Lett. 65, 2221

Ristow, G. H. 1996 Dynamics of granular materials in a rotating drum. Europhys. Lett. 34, 263.

SAVAGE, S. B. 1993 Mechanics of granular flows. In Continuum Mechanics in Environmental Sciences and Geophysics (ed. K. Hutter). CISM No. 337, pp. 467-552. Springer.

Savage, S. B. \& Hutter, K. 1989 The motion of a finite mass of granular material down a rough incline. J. Fluid Mech. 199, 177-215.

Savage, S. B. \& Hutter, K. 1991 The dynamics of avalanches of granular materials from initiation to runout. Part I: Analysis. Acta Mech. 86, 201-223.

Savage, S. B. \& Lun, C. K. K. 1988 Particle size segregation in inclined chute flow of dry cohesionless granular solids. J. Fluid Mech. 189, 311-335.

TAI, Y. C. \& GraY, J. M. N. T. 1998 Limiting stress states in granular avalanches. Annal. Glaciol. 26, 272-276.

Tóth, G. \& ODSTRČIL, D. 1996 Comparison of some flux corrected transport and total variation diminishing numerical schemes for hydrodynamic and magnetohydrodynamic problems. J. Comput. Phys. 128, 82-100.

Ullrich, M. 1969 Entmischungserscheinungen in Kugelschüttungen. Chemie-Ing.-Techn. 41(16), 903-907.

Wieland, M., Gray, J. M. N. T. \& Hutter, K. 1999 Channelised free surface flow of cohesionless granular avalanches in a chute with shallow lateral curvature. J. Fluid Mech. 392, 73-100.

YeE, H. C. 1989 A class of high-resolution explicit and implicit shock-capturing methods. NASA TM-101088. 218pp. 